University of Nebraska - Lincoln

DigitalCommons@University of Nebraska - Lincoln

P. F. (Paul Frazer) Williams Publications

Electrical \& Computer Engineering, Department

May 1976

\title{
Resonance Raman scattering of light from a diatomic molecule
}

D. L. Rousseau

Bell Laboratories, Murray Hill, New Jersey

P. F. Williams

University of Nebraska - Lincoln, pfw@moi.unl.edu

Follow this and additional works at: https://digitalcommons.unl.edu/elecengwilliams

Part of the Electrical and Computer Engineering Commons

Rousseau, D. L. and Williams, P. F., "Resonance Raman scattering of light from a diatomic molecule" (1976). P. F. (Paul Frazer) Williams Publications. 24.

https://digitalcommons.unl.edu/elecengwilliams/24

This Article is brought to you for free and open access by the Electrical \& Computer Engineering, Department of at DigitalCommons@University of Nebraska - Lincoln. It has been accepted for inclusion in P. F. (Paul Frazer) Williams Publications by an authorized administrator of DigitalCommons@University of Nebraska - Lincoln. 


\title{
Resonance Raman scattering of light from a diatomic molecule
}

\author{
D. L. Rousseau \\ Bell Laboratories, Murray Hill, New Jersey 07974
}

P. F. Williams

Bell Laboratories, Murray Hill, New Jersey 07974

and Department of Physics, University of Puerto Rico, Rio Piedras, Puerto Rico 00931

(Received 13 October 1975)

\begin{abstract}
Resonance Raman scattering from a homonuclear diatomic molecule is considered in detail. For convenience, the scattering may be classified into three excitation frequency regions-off-resonance Raman scattering for incident energies well away from resonance with any allowed transitions, discrete resonance Raman scattering for excitation near or in resonance with discrete transitions, and continuum resonance Raman scattering for excitation resonant with continuum transitions, e.g., excitation above a dissociation limit or into a repulsive electronic state. It is shown that the many differences in scattering properties in these three excitation frequency regions may be accounted for by expressions derived from simple perturbation theory. Scattering experiments from molecular iodine are presented which test and verify the general scattering theories. Spectral measurements, time decay measurements, and pressure broadening measurements were made on $\mathrm{I}_{2}$ in the discrete resonance Raman scattering region; and spectral measurements at several excitation frequencies were made in the continuum resonance Raman scattering region. In each case calculations based on general theories correctly describe the experimental data.
\end{abstract}

\section{INTRODUCTION}

Over the past few years a great deal of interest has developed in the understanding of resonance Raman scattering and its application to problems in chemistry, physics, and biology. Most of these studies have been devoted to either solids or complex biological molecules, and relatively little attention has been given to simple gaseous molecules. Resonance Raman scattering is generally not well understood, and the work on solids and complex molecules has done little to clarify the basic mechanisms. In solids, the lack of success may be attributed to complicated electronic structures, impurities, anisotropies, and ill defined electronphonon interactions. Similarly, biological molecules often have strong complex absorption bands; vibrational modes may not be readily assigned; broad fluorescence frequently obscures the discrete structure; and the spectra have a marked excitation frequency dependence. In contrast, the electronic structure of some small molecules are quite simple and very well known. Therefore, the properties of the light scattering from such molecules as the incident laser frequency is varied from nonresonance, to resonance with discrete vibration-rotation levels of an excited state, and then at high laser energies to resonance with continuum states, should serve to elucidate the nature of the fundamental processes. In this paper we discuss the scattering behavior of monochromatic light when the intermediate states are isolated discrete vibrationalrotational levels of a diatomic molecule and the scattering behavior when the intermediate states belong to a continuum.

Empirically, very different scattering spectra are obtained if the incident laser frequency is far from resonance, is in resonance with transitions to discrete states, or is in resonance with transitions to continuum states. The differences between the scattering behavior in these three regions is so great that they have been used to classify ${ }^{1}$ the re-emission into normal Raman scattering, resonance fluorescence, and resonance Raman scattering, respectively. This empirical classification, resulting from the marked differences in the scattering behavior, have led to a popular belief ${ }^{2}$ that fundamental theoretical differences exist between Raman scattering, resonance Raman scattering, and resonance fluorescence. As pointed out recently by Behringer, ${ }^{3,4}$ this problem is not new, but dates back to the turn of the century with the question of the differences between ordinary Rayleigh scattering and resonance Rayleigh scattering. Since this time, two schools of thought have evolved concerning the origins of resonance and nonresonance scattering. The first asserts that the long-lived resonance fluorescence process is a different physical process than short-lived resonance Raman scattering. The distinction between the processes is made by asserting that in resonance fluorescence the excitation and re-emission may be divided into a real absorption followed independently by a real emission. In contrast, it is argued that in $\mathrm{Ra}-$ man scattering (or Rayleigh scattering) this bisection of the elementary processes is impossible. It is consequently concluded that two distinguishably different physical processes account for these phenomena. The other viewpoint is that resonance fluorescence and $\mathrm{Ra}-$ man scattering, including resonance Raman scattering, are all one process with the empirical differences interpretable from the excitation frequency differences alone.

Consideration of the scattering of monochromatic light from simple gaseous diatomic molecules clarifies this question in a definitive way. As long as scattering amplitudes from nearby states are sufficiently small, compared to the resonant state under consideration, ${ }^{5}$ so as to be neglected, and as long as quasielastic collision- 


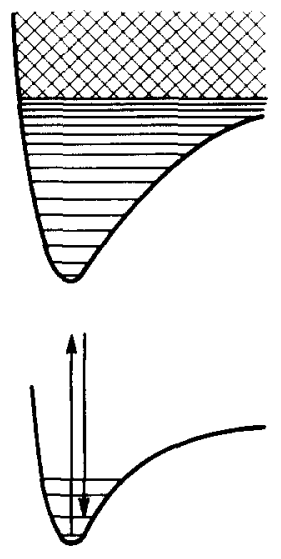

A

\section{RAMAN SCATTERING}

al broadening effects are small, no distinction between resonance fluorescence and resonance Raman scattering may be made. From perturbation theory a general expression may be derived which is valid for scattering of monochromatic light independent of the excitation frequency. The spectrum of scattered light obeys this simple formalism and varies in a predictable systematic way with excitation frequency. From the theory it is clear that even for exact resonance the excitation and the re-emission process are not independent, because the emitted photon retains the phase information of the excitation step.

At exact resonance, the scattering amplitude diverges owing to the pole in the denominator, and a damping term must be included. If we take the relevant matrix elements to be real, then doing so introduces an imaginary component into the scattering amplitude which is strongly peaked at this resonant frequency. It then becomes tempting to classify the real part as resonance Raman scattering and the imaginary part as resonance fluorescence, ${ }^{2}$ This arbitrary separation into ill-defined processes is confusing, however, since each term results from the same perturbation expression. The contributions from each term are most clearly seen in scattering from continuum intermediate states, in which case the real and the imaginary terms contribute about equally to the scattering intensity and no physical differences between the scattering resulting from the two contributions may be made. Furthermore, in considering resonance with a single sharp intermediate state the scattering characteristics vary in a smooth continuous manner according to the Lorentzian line shape function of that state, and the question of where resonance fluorescence ends and resonance Raman scattering begins becomes obviously meaningless.

For ease of discussion we wish to classify the range of laser frequencies into three distinct regions. This classification is illustrated in Fig. 1. On the left, when the incident frequency is far from resonance with a real transition we label the re-emission as normal

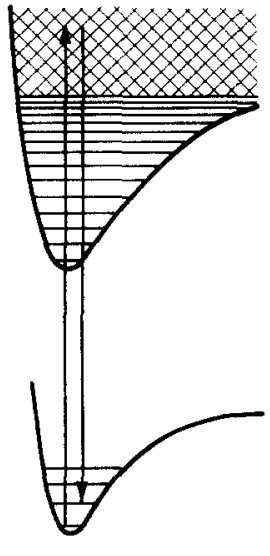

$\mathrm{C}$

CONTINUUM

RESONANCE

RAMAN

SCATTERING
FIG. 1. Classification of Raman scattering according to laser frequency. A. The incident laser frequency is far from resonance with any real electronic transition, so normal Raman scattering is observed. B. The incident laser frequency is in the region of discrete levels of a single electronic intermediate state. We term this process discrete resonance Raman scattering. C. The incident frequency is in the range of a dissociative continuum. We label this process continuum resonance Raman scattering;
Raman scattering. In the center, when the incident frequency is in the region of discrete vibronic transitions we term the re-emission discrete resonance Raman scattering independent of whether or not the incident frequency is at exact resonance. To the right, when the incident frequency is above the excited state dissociation limit in a continuum region, we label the re-emission as continuum resonance Raman scattering.

To illustrate the general light scattering principles discussed in this paper we have selected molecular iodine as an example, $I_{2}$ was chosen for the experiments because it is nearly ideally suited both experimentally and theoretically. First, iodine's electronic structure and the energies of the vibrational-rotational levels are very well known ${ }^{6}$ from fluorescence, predissociation, and photofragmentation experiments. Secondly, iodine is a single isotope, homonuclear diatomic molecule with only a single vibrational degree of freedom. Finally, as illustrated in Fig. 2, the presence of an excited electronic state with a dissociation limit at 20 , $162 \mathrm{~cm}^{-1}$ above the bottom of the ground state well, makes it possible to investigate resonance processes below the dissociation limit (in the discrete resonance Raman scattering region) and above the dissociation limit (in the continuum resonance Raman scattering region) with conventional argon and krypton ion laser s ources.

As was indicated in Fig. 1, analysis of light scattering spectra divides naturally into three distinct regions with very different characteristics. The characteristics ${ }^{1}$ from each of these regions are listed in Table $I$ and discussed in greater detail below.

In the case of ordinary nonresonance Raman scattering, the reradiation is characterized by the following: (1) The Rayleigh line is strong and the fundamental vibrational transition $(\Delta v=1)$ is weak. The higher overtones are weaker yet and are generally not observed at all in the spectrum; (2) The scattered band envelope is relatively broad, may have some structure, 


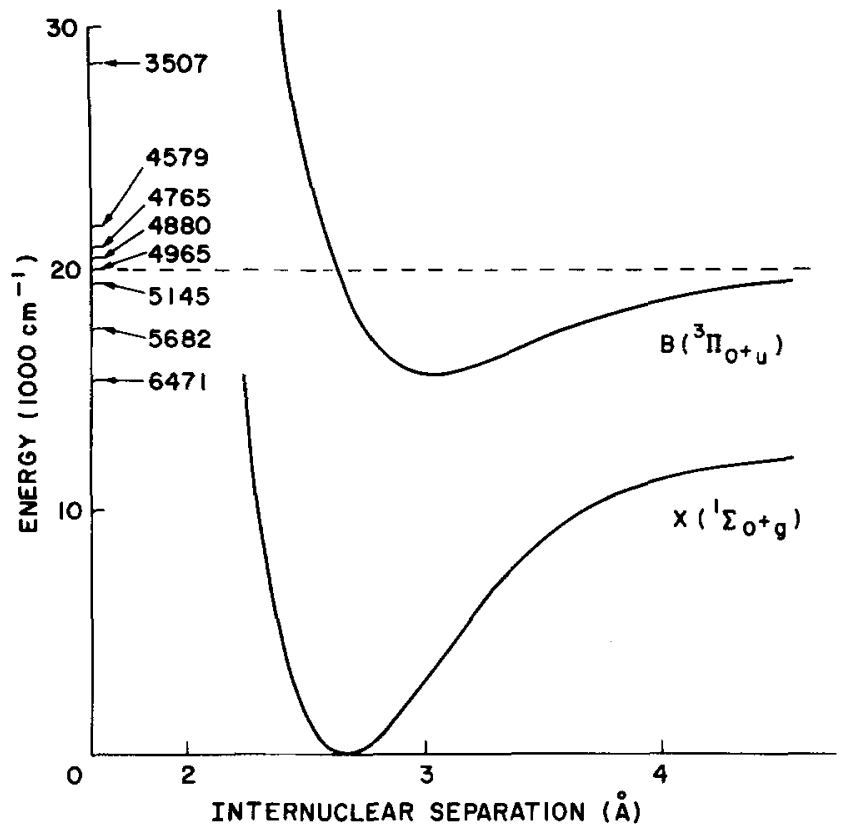

FIG. 2. The $X$ and the $B$ electronic state potential functions of molecular iodine. The dashed line at $20162 \mathrm{~cm}^{-1}$ is the dissociation limit of the $B$ state. The numerical listing on the left corresponds to the excitation energies from $v^{\prime \prime}=0$ of the ground state of the prominent argon and krypton laser lines (expressed in angstroms).

and exhibits no dependence on the excitation frequency; (3) The scattering intensity varies slowly and smoothly (an $\omega^{4}$ dependence) with excitation frequency; (4) The Stokes-anti-Stokes ratio may be calculated from the Boltzmann factor; (5) The scattering time is very fast; (6) Addition of for eign gas does not quench the scattering intensity; (7) The $Q$ branch depolarization ratio is small, with the exact value determined by the anisot- ropy in the polarizability.

At higher incident laser frequencies in the region of discrete resonance Raman scattering the properties of the re-emission are significantly different: (1) The overtones may be of comparable intensity and in fact may be as strong as the Rayleigh line. The ratio of the intensity of one overtone to the next varies erratically; (2) With sufficiently narrow excitation each overtone consists of only a few very sharp lines. Their widths are limited by the combination of the natural, collisional, and Doppler broadening in addition to possible nuclear hyperfine splitting. The structure within each overtone changes erratically with small incident frequency shifts; (3) The scattered intensity varies wildly with excitation frequency; (4) The Stokes-anti-Stokes ratio is not simply related to the ground state population factors and in fact the anti-Stokes intensity may be greater than the Stokes intensity in some cases; (5) At exact resonance the scattering time is long and is typically in the range of $10^{-5}-10^{-8} \mathrm{sec}$, although as the incident frequency is moved away from exact resonance the scattering time becomes very short; (6) Addition of foreign gas strongly quenches the intensity; (7) Generally the scattering of the $Q$ branch is depolarized, with a depolarization ratio of $\frac{3}{4}$.

In the region of continuum resonance Raman scattering the observed spectra are substantially different from those in the discrete resonance case: (1) Again there are numerous overtones with comparable intensity to the fundamental and the Rayleigh line. The intensity variation from one overtone to the next is now very regular; (2) The band envelope within each overtone region appears broad with considerable structure which smoothly changes as the excitation frequency is varied; (3) The scattering intensity varies with the excitation frequency in a smooth systematic way; (4)

TABLE I. Excitation-frequency dependent characteristics of light scattering from diatomic molecules.

\begin{tabular}{|c|c|c|c|}
\hline Characteristics & $\begin{array}{l}\text { Nonresonance } \\
\text { Raman scattering }\end{array}$ & $\begin{array}{l}\text { Discrete resonance } \\
\text { Raman scattering }\end{array}$ & $\begin{array}{l}\text { Continuum resonance } \\
\text { Raman scattering }\end{array}$ \\
\hline $\begin{array}{l}\text { 1. Overtone } \\
\text { intensity }\end{array}$ & No overtones & $\begin{array}{l}\text { Strong; irregular } \\
\text { variation }\end{array}$ & $\begin{array}{l}\text { Strong; systematic } \\
\text { variation }\end{array}$ \\
\hline $\begin{array}{l}\text { 2. Band } \\
\text { envelope }\end{array}$ & $\begin{array}{l}\text { Broad; structured; } \\
\text { no frequency } \\
\text { dependence }\end{array}$ & $\begin{array}{l}\text { Sharp; erratic } \\
\text { frequency dependence }\end{array}$ & $\begin{array}{l}\text { Broad; structured; } \\
\text { systematic frequency } \\
\text { dependence }\end{array}$ \\
\hline $\begin{array}{l}\text { 3. Frequency } \\
\text { dependence } \\
\text { of intensity }\end{array}$ & $\omega_{S}^{4}$ & Strong and erratic & Strong and systematic \\
\hline $\begin{array}{l}\text { 4. Stokes-anti- } \\
\text { Stokes ratio }\end{array}$ & $\propto$ Boltzman factor & $\begin{array}{l}\text { Not solely population } \\
\text { dependent }\end{array}$ & $\begin{array}{l}\text { Not solely population } \\
\text { dependent }\end{array}$ \\
\hline $\begin{array}{l}\text { 5. Scattering } \\
\text { time }\end{array}$ & $\begin{array}{l}\text { Short } \leqslant 10^{-14}-10^{-15} \\
\text { sec }\end{array}$ & $\begin{array}{l}\text { Variable } \sim 10^{-5}-10^{-12} \\
\text { sec }\end{array}$ & Short $\leqslant 10^{-12} \mathrm{sec}$ \\
\hline $\begin{array}{l}\text { 6. Quenching } \\
\text { behavior }\end{array}$ & Unquenched & Quenched & Unquenched \\
\hline $\begin{array}{l}\text { 7. Q-branch } \\
\text { depolariza- } \\
\text { tion ratio }\end{array}$ & Less than $\frac{3}{4}$ & $\frac{3}{4}$ & Less than $\frac{3}{4}$ \\
\hline
\end{tabular}


The Stokes-anti-Stokes ratio is not solely related to the ground state population factors but varies systematically with incident frequency; (5) The scattering time is fast; (6) Addition of foreign gas does not quench the intensity; (7) The depolarization ratio is small and may be calculated from the electronic structure of the molecule.

These disparate properties may be interpreted by using standard second order perturbation theory. In the next section the relevant expressions are derived and in the following sections we use these results to discuss the interpretation of the properties listed here. We also present data obtained on molecular iodine which tests the validity of several aspects of the formalism.

\section{THEORY}

The total scattering intensity $I_{s}$ in photons per molecule per second for a transition from the initial ground state, $|G\rangle$, to the final state, $|F\rangle$, may be given by the standard expression 7,8

$$
I_{s}=\frac{8 \pi \omega_{s}^{4} I_{L}}{9 c^{4}} \sum_{\rho \sigma}\left|\left(\alpha_{\rho \sigma}\right)_{G F}\right|^{2} .
$$

Here $c$ is the velocity of light, $I_{L}$ is the incident intensity at frequency $\omega_{L}$, and $\omega_{s}$ is the scattered frequency. $\left(\alpha_{\rho \sigma}\right)_{G F}$ is the polarizability tensor for the transition from $|G\rangle$ to $|F\rangle$ with incident and scattered polarizations indicated by $\sigma$ and $\rho$, respectively. The second order perturbation expression for $\left(\alpha_{\rho \sigma}\right)_{G F}$ is

$$
\begin{aligned}
\left(\alpha_{\rho \sigma}\right)_{G F}=\frac{1}{\hbar} \sum_{I} & \left(\frac{\left\langle F\left|p_{\rho}\right| I\right\rangle\left\langle I\left|p_{\sigma}\right| G\right\rangle}{\omega_{G I}-\omega_{L}}\right. \\
& \left.+\frac{\left\langle I\left|p_{\rho}\right| G\right\rangle\left\langle F\left|p_{\sigma}\right| I\right\rangle}{\omega_{I F}+\omega_{L}}\right) .
\end{aligned}
$$

In this expression $\omega_{G I}$ is the energy spacing between an excited rovibronic state $|I\rangle$ and the ground state $|G\rangle$; and $p$ is the electron momentum operator. For the subsequent discussion we will neglect the second term in Eq. (2), since near resonance it is small in comparison to the first term owing to its large resonance denominator. In addition, for simplicity we will neglect the polarization subscripts. It must be noted that Eq. (2) is completely general and is expected to correctly treat both Raman and fluorescent processes within the limits of pertrubation theory. However, it is very difficult to make meaningful interpretations or calculations from it because the states $|F\rangle,|G\rangle$, and $|I\rangle$ must be described by complete rovibronic wavefunctions which depend on both nuclear and electronic coordinates. A variety of approximations and expansions are therefore made at this point, ${ }^{2,8,9}$ after which the basic physical processes often become obscured. In the discussion presented here we have adopted a very simplistic approach, but one which makes very transparent the frequency dependence of the light scattering. This general approach and other more sophisticated ones may be found in a variety of more lengthy discussions of the theories of Raman and resonance Raman scattering. ${ }^{7,8}$

In order to make Eq. (2) more manageable ${ }^{7}$ it is first convenient to separate out the vibrational wavefunction by using the Born-Oppenheimer approximation,

$$
\psi_{e, v}=\theta_{e}(r, R) \varphi_{e, v}(R) .
$$

Here $\psi_{e, v}$ is the total vibronic wavefunction; $\theta_{e}(r, R)$ is the electronic wavefunction (with electronic quantum number $e$ ) which depends on the electron coordinates $r$ and the nuclear coordinates $R . \varphi_{e, v}(R)$ is a vibrational wavefunction of electronic state $e$ with vibrational quantum number $v$.

In writing Eq. (3) in this form we implicitly ignore the dependence of the wavefunctions on molecular orientation (the angular coordinates of $\mathbf{R}$ ). Inclusion of this dependence in the matrix elements of Eq. (2) only results in a multiplicative factor which depends on the rotational quantum numbers and on the polarization of the incident and scattered light. This factor is responsible for the dipole selection rule on rotational transitions, but in most of the following discussion it plays no other role and for clarity of presentation it will be ignored. In the discussion of continuum resonance Raman scattering we will want to compare intensities of lines with different rotational quantum numbers, and in Eq. (26) this rotational factor reappears as the factor $b_{J}$. Detailed evaluation of these rotational matrix elements has been carried out most recently by Silverstein and St. Peters ${ }^{10}$ and by Jacon and Van Labeke. ${ }^{11}$

By substituting Eq. (3) into Eq. (2) we may rewrite the matrix elements as follows,

$\langle F|p| I\rangle=\left\langle\varphi_{e_{F}, f}(R) \theta_{e_{F}}(r, R)|p| \theta_{e_{I}}(r, R) \varphi_{e_{I}, i}(R)\right\rangle$,

in which expression the electronic matrix element is

$$
M(R)=\left\langle\theta_{e_{F}}(r, R)|p| \theta_{e_{I}}(r, R)\right\rangle .
$$

The electronic matrix elements, such as those shown here, are expected to be weakly varying functions of the internuclear separation $R$, so they may be expanded in a rapidly converging Taylor series,

$$
M(\xi)=M\left(\xi_{0}\right)+\left(\frac{\partial M}{\partial \xi}\right)_{\xi_{0}} \Delta \xi+\cdots \cdot
$$

In this equation $M\left(\xi_{0}\right)$ is the electronic matrix element evaluated at the equilibrium internuclear separation $\xi_{0}$, and $\Delta \xi$ is the deviation from this separation. For simplicity, the expansion was carried out in a dimensionless variable $\xi$ where

$$
\xi=R\left(\frac{\mu \omega_{0}}{\hbar}\right)^{1 / 2} \text {. }
$$

$\mu$ is the reduced mass of the oscillator and $\omega_{0}$ its harmonic frequency.

Using the Taylor expansion we obtain for the zeroth and 1 st order terms in the perturbation series

$$
\begin{aligned}
(\alpha)_{g f}= & {\left[M\left(\xi_{0}\right)\right]^{2} \frac{1}{\hbar} \sum_{i} \frac{\langle f \mid i\rangle\langle i \mid g\rangle}{\omega_{g i}-\omega_{L}}+M\left(\xi_{0}\right)\left(\frac{\partial M}{\partial \xi}\right)_{\xi_{0}} } \\
& \times \frac{1}{\hbar} \sum_{i} \frac{\langle f|\Delta \xi| i\rangle\langle i \mid g\rangle+\langle f \mid i\rangle\langle i|\Delta \xi| g\rangle}{\omega_{g i}-\omega_{L}}+\cdots .
\end{aligned}
$$

Now the states $|g\rangle$ and $|f\rangle$ correspond to vibrational 
wavefunctions of the ground electronic state and $|i\rangle$ to the vibrational wavefunctions of the excited electronic state. For simplicity, we now are considering only a single excited electronic state. It should be pointed out that the matrix elements of the type $\langle f \mid i\rangle$, etc., correspond to Franck-Condon overlap amplitudes. To understand the expected intensities in a light scattering experiment we must determine the magnitude of the two terms in Eq. (8) in various limits.

\section{A. Normal Raman scattering}

First let us consider the condition ${ }^{7}$ that the incident laser energy is very much less than the energy $\omega_{g i}$ needed for a real transition, i. e., $\omega_{L} \ll \omega_{B i}$. This represents off-resonance light scattering as indicated in diagram $\mathrm{A}$ of Fig. 1. Because of the above inequality the denominator in both terms may be considered nearly a constant, $\Omega=\omega_{g i}-\omega_{L}$, and the sum over intermediate states may then be contracted using completeness to give

$$
\begin{aligned}
(\alpha)_{g f}= & {\left[M\left(\xi_{0}\right)\right]^{2} \frac{1}{\hbar \Omega}\langle f \mid g\rangle } \\
& +M\left(\xi_{0}\right)\left(\frac{\partial M}{\partial \xi}\right)_{\xi_{0}} \frac{2}{\hbar \Omega}\langle f|\Delta \xi| g\rangle+\cdots .
\end{aligned}
$$

The vibrational wavefunctions are orthogonal so the matrix element in the first term gives a Kronecker delta function in the inital and final vibrational quantum numbers, $v_{g}^{\prime \prime}, v_{f}^{\prime \prime}$, and if we assume the ground electronic potential is harmonic the second term vanishes unless $v_{f}^{\prime \prime}=v_{g}^{\prime \prime} \pm 1$. Therefore, for off-resonance light scattering

$$
\begin{aligned}
(\alpha)_{g f}= & {\left[M\left(\xi_{0}\right)\right]^{2} \frac{1}{\hbar \Omega} \delta_{v_{g}^{\prime \prime}, v_{f}^{\prime \prime}} } \\
& +M\left(\xi_{0}\right)\left(\frac{\partial M}{\partial \xi}\right)_{\varepsilon_{0}} \frac{2}{\hbar \Omega}(Z)_{v_{g}^{\prime \prime}, v_{f}^{\prime \prime}}+\cdots,
\end{aligned}
$$

where

$$
\begin{aligned}
Z & =0 & & \text { if } v_{g}^{\prime \prime} \neq v_{f}^{\prime \prime} \pm 1 \\
& =\left[\left(v_{g}^{\prime \prime}+1\right) / 2\right]^{1 / 2} & & \text { if } v_{g}^{\prime \prime}=v_{f}^{\prime \prime}+1 \\
& =\left(v_{g}^{\prime \prime} / 2\right)^{1 / 2} & & \text { if } v_{g}^{\prime \prime}=v_{f}^{\prime \prime}-1 .
\end{aligned}
$$

We see from Eq. (10) that the first term results in unshifted scattered radiation (Rayleigh scattering) and the second term gives re-emission shifted from the incident photon by plus or minus one vibrational quantum, hence Raman scattering at the fundamental frequency. Because the Taylor series is rapidly converging, the Raman term is expected to be very much weaker than the Rayleigh term, and higher order terms in the expansion which result in overtones in the Raman spectrum are expected to be weaker yet. Indeed, from a comparison of the Rayleigh to Raman scattering cross sections in simple gases it appears that the experimental ratio of intensities of the coefficients of the two terms in Eq. (10) is typically about 3 orders of magnitude.

The weak variation of the energy denominator in Eq. (8) may be treated by expanding it in a Taylor series about some average frequency, $\Omega$. Doing so results in the addition of another term in Eq. (9) proportional to $\langle f|\Delta \xi| g\rangle$. This term may be significant and should be included in any detailed study of off-resonant Raman scattering, but its inclusion leaves the predicted qualitative behavior of the scattering unchanged-the Rayleigh line is much more intense than the fundamental, etc. Since we are primarily interested in resonant scattering, for which case all approximations based on a slowly varying denominator fail completely anyway, a more complete discussion of these approximations would lead us too far afield and we refer the reader to Ref. 8, where a complete discussion is presented.

\section{B. Discrete resonance Raman scattering}

\section{Frequency spectrum}

In the limit of the laser frequency coinciding with a real transition $\left(\omega_{L} \approx \omega_{g i}\right)$ for some $|i\rangle$ independent of whether or not the levels are discrete or continuous, a situation completely different from the nonresonance case arises. In this resonance condition in the sum over states in $\mathrm{Fq}$. (8) in no approximation whatsoever are the denominators constant, so the completeness argument fails and all terms may contribute to both the Rayleigh and the Raman scattering. However, because of the rapidly converging Taylor series in which the coefficient of the second term in typically about 3 orders of magnitude smaller than the first, most of the Rayleigh and the Raman scattering intensity will now result from the first term alone i.e.

$$
(\alpha)_{g f}=\left[M\left(\xi_{0}\right)\right]^{2} \frac{1}{\hbar} \sum_{i} \frac{\langle f \mid i\rangle\langle i \mid g\rangle}{\omega_{g i}-\omega_{L}} .
$$

No longer is there a selection rule discriminating against overtones, but instead the overtones are expected to be strong and dependent only on the magnitude of the appropriate Franck-Condon overlap factors.

Further simplifications of Eq. (11) may be made for discrete resonance Raman scattering. When the incident frequency is in the discrete resonance region and resonant with a specific initial to intermediate state transition then that transition should dominate the summation and all other transitions are weak in comparison. Equation (11) may then be written

$$
(\alpha)_{g f}=\left[M\left(\xi_{0}\right)\right]^{2} \frac{1}{\hbar} \frac{\langle f \mid i\rangle\langle i \mid g\rangle}{\left(\omega_{g i}-\omega_{L}-i \Gamma\right)}
$$

Note that because we are now considering resonance with a single discrete state, it is necessary to include the damping term, $i \Gamma$, which was omitted from the previous formulas for simplicity. The linewidth, $\Gamma$, was of course not important in the off-resonance $\mathrm{Ra}$ man case because it was so small in comparison to $\omega_{g t}-\omega_{L}$. From Eq. (1) we then obtain the following expression for the scattering intensity:

$$
I_{S}=\frac{8 \pi \omega_{s}^{4} I_{L}}{9 c^{4} \hbar^{2}}\left[M\left(\xi_{0}\right)\right]^{4} \frac{|\langle f \mid i\rangle\langle i \mid g\rangle|^{2}}{\left(\omega_{g i}-\omega_{L}\right)^{2}+\Gamma^{2}} 。
$$

From this equation we see that the structure in the spectrum of the re-emitted light then will depend on the specific vibrational and rotational quantum numbers of the selected excited state. The intensities of the successive overtones will vary erratically depending on the 
magnitudes of the Franck-Condon overlap factors connecting the intermediate and final states. A slight change in incident frequency will bring about resonance with a different discrete state of the complicated excited state vibration-rotational manifold, with different vibrational and rotational quantum numbers so that a completely different appearing re-emission spectrum may be observed. The effect of collisional energy redistribution further complicates this spectrum as will be seen later.

\section{Re-emission lifetime}

While the above discussion described the frequency dependence of the intensity variations for discrete Raman scattering, time dependent excitation intensities $^{12}$ are needed to understand the re-emission lifetime behavior. Consider a simple three level system such as that shown in Fig. 3. The scattering process consists of the absorption of an excitation photon (frequency $\omega_{L}$ ) and the emission of a Raman shifted photon (frequency $\omega_{s}$ ) with a consequent change in the system state from $|g\rangle$ to $|f\rangle$. Although throughout this section we use Raman scattering as our model in the lifetime calculations, the arguments are equally applicable to Rayleigh scattering. We now apply a pulse of light, such as that shown in Fig. 4, of frequency $\omega_{L}$ to the system and ask what the time response of the Raman shifted re-emission is. For the moment we consider arbitrary pulse shapes, with the only restriction being that the pulse intensity is zero for $t<0$. For weak excitation fields, the time response of the Raman shifted radiation may be determined by treating the excitation pulse as a perturbation on the system and using second order time dependent perturbation theory to calculate the probability amplitude for the system to be in state $|f\rangle$, with the emission of a Raman photon $\left(\omega_{s}\right)$, and the absorption of a laser photon $\left(\omega_{L}\right)$ at time $t$ given that at $t=0$ it was in $|g\rangle$. This amplitude will be denoted by $\left\langle f ; \omega_{s} \mid \psi_{2}(t)\right\rangle$, where $\omega_{s}$ is the frequency of the re-emitted Raman photon, and the subscript 2 indicates that the state $|\psi\rangle$ is correct to second order. If the re-emission is observed with a spectrometer with frequency resolution function $g(\omega)$, then the observed intensity of the Raman shifted radiation has the following proportionality:

$$
I_{s}(t) \propto \frac{d}{d t} \int_{0}^{\infty} g\left(\omega_{s}\right)\left|\left\langle f ; \omega_{s} \mid \psi_{2}(t)\right\rangle\right|^{2} d \omega_{s} .
$$

In order to eliminate distortions of the time response

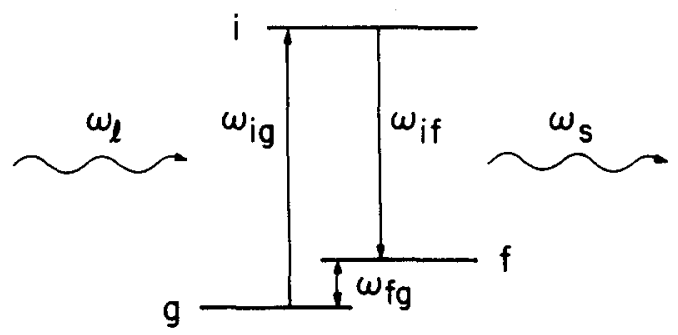

FIG. 3. Model three level system for considering discrete resonance Raman scattering. $\omega_{L}$ and $\omega_{S}$ are the incident and scattered frequencies, respectively. (a)

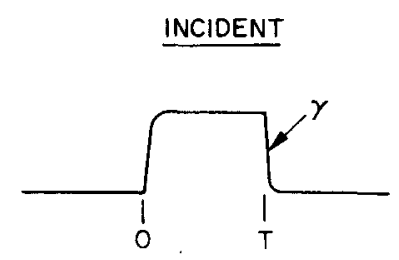

(b)

SCATTERED
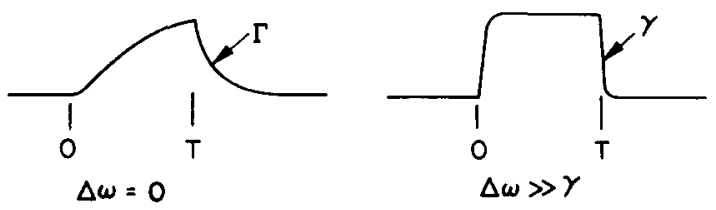

FIG. 4. Incident and scattered theoretical pulse shapes. a. Rectangular laser pulse with finite rise and fall times used in the calculations, b. Calculated scattered pulse shapes for resonant excitation $(\Delta \omega=0)$ and off-resonant excitation $(\Delta \omega \gg \gamma)$.

of the Raman intensity caused by using too narrow a frequency filter, the spectrometer frequency resolution is taken to be sufficiently broad that it passes essentially all of the Raman reradiation. Doing so, we may set $g(\omega)=1$.

If the excitation field is given by $E_{L}(t)=e^{i \omega_{L} t} S(t)$, where $S(t)=0$ for $t \leq 0$, then the second order transition amplitude may be written

$$
\left\langle f ; \omega_{s} \mid \psi_{2}(t)\right\rangle=\frac{1}{\hbar^{2}}\langle f|p| i\rangle\langle i|p| g\rangle F(t),
$$

where

$$
\begin{aligned}
F(t)= & e^{-i \omega_{f g} t} \int_{0}^{t} d \tau \int_{0}^{\tau} d \tau^{\prime} e^{-i\left(\omega_{i f}-i \Gamma\right) \tau} e^{i \omega_{s} \tau} e^{i\left(\omega_{i g}-i \Gamma\right) \tau^{\prime}} \\
& \times e^{-i \omega_{L} \tau^{\prime}} S\left(\tau^{\prime}\right)
\end{aligned}
$$

Here $\omega_{f g}$ and $\omega_{i g}$ are the energies of the states $|f\rangle$ and $|i\rangle$, relative to $|g\rangle ;(2 \Gamma)^{-1}$ is the natural lifetime of the state $|i\rangle$; and the variables of integration, $\tau, \tau^{\prime}$, correspond, respectively, to the time of absorption of a laser photon and the time of re-emission of a Raman photon.

Although the algebra is somewhat tedious, the integral in Eq. (15) may be carried out and Eq. (14) evaluated. It is simpler, however, to note ${ }^{13}$ that the time dependence of the re-emitted light intensity is determined by the time evolution of the population of the intermediate state, i. e.,

$$
\begin{aligned}
I_{s}(t) & \propto \frac{d}{d t} \int_{0}^{\infty}\left|\left\langle f ; \omega_{s} \mid \psi_{2}(t)\right\rangle\right|^{2} d \omega_{s} \\
& =\frac{2 \pi}{\hbar^{2}}|\langle f|p| i\rangle|^{2}\left|\left\langle i \mid \psi_{1}(t)\right\rangle\right|^{2},
\end{aligned}
$$

where $\left|\left\langle i \mid \psi_{1}(t)\right\rangle\right|^{2}$ is the admixture of the intermediate state $|i\rangle$ into the state $|g\rangle$ by the perturbation, $E_{L}(t)$, correct to first order. In analogy with the preceding discussion, this quantity is determined by 


$$
\left\langle i \mid \psi_{1}(t)\right\rangle=\frac{1}{\hbar}\langle i|p| g\rangle G(t)
$$

where

$$
G(t)=e^{-\Gamma t} e^{-i \omega_{i} t} \int_{0}^{t} e^{i\left(\omega_{i}-i \Gamma\right) \tau} e^{-i \omega_{L} \tau} S(\tau) d \tau .
$$

Using Eq. (17) in Eq. (16), we obtain for the observed Raman intensity

$$
I_{s}(t)=\frac{8 \pi \omega_{s}^{4} I_{L}}{9 c^{4} \hbar^{2}}|\langle f|p| i\rangle\langle i|\phi| g\rangle|^{2}|G(t)|^{2}
$$

Upon specification of the pulse envelope function, $S(t)$, the evaluation of $G(t)$ proceeds simply. For clarity of discussion, we take pulse to be as shown in Fig. 4. Finite exponential rise and fall times are assumed in order to minimize the effects of transients.
Using the above formulation, other pulse shapes such as a cusp function, may be handled with equal facility. We have chosen the shape shown in Fig. 4 because it does not require the excitation to have been turned on for an infinite time in the past, and since by choosing $T$ sufficiently large, the deconvolution of the input pulse from the output is relatively easy.

Specifically, the function $S(t)$ is defined by

$$
\begin{aligned}
S(t) & =0 & & t \leq 0 \\
& =\left(1-e^{-\gamma t}\right) & & 0 \leq t \leq T \\
& =\left(1-e^{-\gamma T}\right) e^{-\gamma(t-T)} & & T \leq t,
\end{aligned}
$$

where $T$ is the pulsewidth, and $\gamma^{-1}$ gives the rise and fall time of the electric field of the pulse. Letting $\Delta \omega$ $=\omega_{i g}-\omega_{L}$ and $\beta=\gamma-\Gamma$, the general result is

$$
\begin{aligned}
&|G(t)|^{2}=\frac{1}{\left(\Delta \omega^{2}+\Gamma^{2}\right)\left(\Delta \omega^{2}+\beta^{2}\right)}\left\{\gamma^{2}\left(1-2 e^{-\Gamma t} \cos \Delta \omega t+e^{-2 \Gamma t}\right)+\left(\Delta \omega^{2}+\Gamma^{2}\right)\left(1-e^{-\gamma t}\right)^{2}\right. \\
&\left.-2 \gamma \Gamma\left(1-e^{-\gamma t}\right)\left[1-e^{-\Gamma t}\left(\cos \Delta \omega t-\frac{\Delta \omega}{\Gamma} \sin \Delta \omega t\right)\right]\right\} \quad 0 \leq t \leq T \\
&=\frac{1}{\left(\Delta \omega^{2}+\Gamma^{2}\right)\left(\Delta \omega^{2}+\beta^{2}\right)}\left\{\gamma^{2}\left(1-2 e^{-\Gamma T} \cos \Delta \omega T+e^{-2 \Gamma T}\right) e^{-2 \Gamma(t-T)}+\left(\Delta \omega^{2}+\Gamma^{2}\right)\left(1-e^{-\gamma T}\right)^{2} e^{-2 \gamma(t-T)}+2 \gamma \Gamma\left(1-e^{-\gamma T}\right) e^{-\gamma(t-T)}\right. \\
&\left.\times\left[e^{-\Gamma t} \cos \Delta \omega t-e^{-\Gamma(t-T)} \cos \Delta \omega(t-T)-\frac{\Delta \omega}{\Gamma}\left(e^{-\Gamma t} \sin \Delta \omega t-e^{-\Gamma(t-T)} \sin \Delta \omega(t-T)\right)\right]\right\} \quad t \geq T \cdot \quad(20)
\end{aligned}
$$

Putting Eq. (20) into Eq. (18), the time dependence of the re-emission is determined.

To obtain a qualitative understanding of the behavior of $|G(t)|^{2}$, it is desirable to take the excitation pulse rise and fall times to be much faster than the natural lifetime of the excited state, that is, $\gamma \gg \Gamma$. Under this condition for exact resonance, $\Delta \omega=\omega_{i g}-\omega_{L}$ $=0$, we get

$$
\begin{aligned}
|G(t)|^{2} & \cong \frac{1}{\Gamma^{2}}\left(1-e^{-\Gamma t}\right)^{2} & & 0 \leq t \leq T \\
& \cong \frac{1}{\Gamma^{2}}\left(1-e^{-\Gamma T}\right)^{2} e^{-2 \Gamma(t-T)} & & T \leq t .
\end{aligned}
$$

$|G(t)|^{2}$ for this case, as shown in Fig. 4, has a rise and fall time characteristic of the natural response time of the system $(2 \Gamma)^{-1}$. However, far off resonance, the response is "fast" in that it follows the excitation pulse, as may also be seen from Eq. (20) by taking the case in which the shift from resonance is much greater than the Fourier broadening of the pulse, $i_{o}$ e., $\Delta \omega \gg \gamma_{\text {。 }}$ Then,

$$
\begin{array}{rlrl}
|G(t)|^{2} & \cong \frac{1}{\Delta \omega^{2}}\left(1-e^{-\gamma t}\right)^{2} & 0 \leq t \leq T \\
& \cong \frac{1}{\Delta \omega^{2}}\left(1-e^{-\gamma T}\right)^{2} e^{-2 \gamma(t-T)} & & T \leq t .
\end{array}
$$

The behavior of $|G(t)|^{2}$ under this condition is also shown in Fig. 4. The case for $\Delta \omega \approx \gamma$ is more complicated because the behavior is, in this case, determined by the rapidly oscillating sin and cos terms in Eq. (20).
From Fqs. (21) and (22) we find that on resonance the time response is "slow, " being limited by the natural lifetime of the excited state. Off resonance, the time response is fast, being limited by the temporal line shape of the probing pulse. The question of what the scattering time is in the intermediate region is an interesting one. Intuitively, we would expect the lifetime to be governed by the uncertainty principle. In this argument the lifetime $\Delta t$ for the re-emission is expected to be limited by the frequency difference $\Delta \omega$ between the excited state transition frequency and the incident frequency, i, e., $\Delta t=1 / \Delta \omega$. That is, if energy is not conserved in the transition to the excited state, by an amount $\Delta \omega$, then the time the molecule can spend in the state if limited to $1 / \Delta \omega$. Unfortunately, efforts to measure such a scattering time are complicated because the precise definition of the scattering time becomes unclear owing to limitations imposed by the frequency-time uncertainty principle.

In order to measure a scattering time $\tau$ in an offresonance experiment, we must sharpen the rise and fall times $(2 \gamma)^{-1}$ of our excitation pulse so that $\tau \gtrsim(2 \gamma)^{-1}$. If we expect a scattering time $\tau$ of order $1 / \Delta \omega$, then this requires that $\Delta \omega \leq \gamma$. In this region $|G(t)|^{2}$ as given by F.q. (20) is rather complicated, with a strong oscillatory component at frequency $\Delta \omega$. The leading and trailing edges of the response pulse are by no means exponential, so that an unambiguous measurement of the scattering time is impossible. The cause of difficulty is easy to uncover. By temporally sharpening our ex- 
citation pulse, we have broadened its frequency spectrum by an amount $\gamma \gtrsim \Delta \omega$. We now see re-emission from a range of excitation frequency components, some of which are resonant with the initial transition. The oscillatory behavior results from an interference of each of these re-emission components. Because even in physically allowable "gedanken" experiments a direct measurement of the scattering time fails, ${ }^{14}$ the question of the detailed nature of its variation with incident frequency must be approached with some care. We can say that on resonance $(\Delta \omega=0)$, the scattering time $\tau$ for the Raman process is equal to the natural lifetime of the excited intermediate state involved, and off resonance, $\tau \leqslant 1 / \Delta \omega$. As we will discuss later, this prediction is completely consistent with all of our experimental results.

\section{Collision effects}

The frequency behavior of the scattering of monochromatic light in situations where collisions are important was derived first by Huber ${ }^{15}$ and later by Mollow and Omont et $a l .,{ }^{16}$ and the theory for the temporal behavior under such collisions was derived by Huber. ${ }^{17}$ In the derivation of the expected spectral re-emission properties that take place when molecular collisions may occur, ${ }^{15}$ the collisional processes were treated in the impact approximation. We obtain for the Raman scattering intensity ${ }^{15,16}$

$$
I_{s}=\frac{8 \pi \omega_{s}^{4} I_{L}}{9 c^{4} \hbar^{2}}=\frac{|\langle f|p| i\rangle|^{2}|\langle i|p| g\rangle|^{2}}{\left[\left(\omega_{L}-\omega_{i g}\right)^{2}+\left(\gamma_{N}+\gamma_{I}+\gamma_{E}\right)^{2}\right]}\left\{\delta\left(\omega_{L}-\omega_{s}-\omega_{f E}\right)+\frac{\gamma_{E}\left(\gamma_{N}+\gamma_{I}+\gamma_{E}\right)}{\pi\left(\gamma_{N}+\gamma_{I}\right)\left[\left(\omega_{s}-\omega_{i f}\right)^{2}+\left(\gamma_{N}+\gamma_{I}+\gamma_{E}\right)^{2}\right]}\right\}
$$

We have neglected Doppler broadening and broadening of the ground and final states. The intermediate state linewidth consists of three Lorentzian contributions: $\gamma_{N}$ corresponds to the natural lifetime; $\gamma_{I}$ is the broadening resulting from a shorter lifetime caused by inelastic collisions, i.e., those collisions in which the molecule is knocked into a different quantum state; and $\gamma_{E}$ is the contribution from quasielastic collisions, i. e., those collisions in which the phase of the oscillator is interrupted but it remains in the same quantum state. Note that the first term in braces in this equation is equivalent to our previously derived Eq. (13).

In the limit of a very low pressure gas $\gamma_{E}$ and $\gamma_{I}$ are zero, so the second term in the braces in Eq. (23) vanishes. In this low pressure limit, and where only one intermediate state is contributing to the scattering amplitude, the first term in Eq. (23) fully characterizes the light scattering. There is a phase memory between the incident and scattered radiation and for an individual molecule the scattering process is coherent regardless of whether or not the incident frequency coincides with the center frequency of the transition. When the incident frequency is moved away from resonance, the intensity of the scattered light varies in a Lorentzian manner as defined by Eq. (23) and may be appropriately termed Raman scattering (resonance Raman scattering for exact resonance).

As the gas pressure is increased, the second term in the braces in Eq. (23) becomes important. This term represents a physically distinguishable process, resulting from a disturbance in the excited state such that the phase is randomized. The re-emission intensity for this incoherent process compared to the Raman process is given by the probability for elastic collisional broadening divided by the probability for radiative re-emission without suffering an elastic collision, i.e., $\gamma_{E} /\left(\gamma_{N}+\gamma_{I}\right)$. Since all phase information is lost in the collision, in contrast to the Raman term this term corresponds to independent absorption and emission processes. The emission resulting from the second term in Eq. (23) is redistributed over the full width of the pressure broadened excited state, and independent of the excitation frequency, it is centered at the resonant energy and has a lifetime of $1 /\left(\gamma_{N}+\gamma_{I}\right)$. Obviously this may occur only if the line broadening mechanism includes a relaxation process which can take up or remove energy. In gases this occurs through quasielastic collisional broadening where during a collision the phase of the oscillator is interrupted but the molecule remains in the same quantum state. Because this term results in a redistribution of the incident energy, to maintain generality we shall refer to it as the redistribution term. We emphasize that the redistribution scattering requires the presence of some excited state relaxation process. In the absence of such a process only the Raman scattering remains, even for exactly resonant excitation. Simple energy conservation arguments then require the re-emission linewidth to be independent of the excited state linewidth. A similar derivation using density matrix formalism was carried out by Shen ${ }^{18}$ for solids and in Ref. 19, where the assumption was made that the ground state linewidths were very narrow, and an expression equivalent to Eq. (23) was obtained.

The temporal response of this term under pulsed excitation was investigated theoretically by Huber ${ }^{17}$ and a general expression was derived describing the time dependence of the intensity. Evaluating his expression (Eq. 3.11 from Ref. 17) for slightly offresonance excitation, we obtain a time decay in the limit of negligible collisional broadening effects of $e^{-\gamma t}$ consistent with our previous result. This result was obtained by assuming negligible collisional broadening and that the natural lifetime $\Gamma$ is significantly longer than the decay time of the excitation pulse $\gamma_{\text {o }}$ If, on the other hand, the collision effects are large, we obtain a time decay of $e^{-\Gamma t}$. We see then that the contribution to the time decay of those molecules unaffected by collisions is one that follows the laser pulse, while the contribution from those that have un- 
dergone pseudoelastic collisions has the time response of the intermediate state.

\section{Continuum resonance Raman scattering}

For excitation into the continuum, ${ }^{20}$ just as in the case of discrete resonance Raman scattering, we must consider Eq. (11):

$$
(\alpha)_{g f}=\left[M\left(\xi_{0}\right)\right]^{2} \frac{1}{\hbar} \sum_{S} \frac{\langle f \mid i\rangle\langle i \mid g\rangle}{\omega_{g i}-\omega_{L}},
$$

and again we would expect overtones to be strong and depend only on the magnitude of the appropriate Franck-Condon factors. Here, because we are dealing with a continuum, we may assume the limit of infinitely narrow states and it is not necessary to include the damping term. However, as will be discussed later, it is necessary to somehow accommodate the pole which occurs for those continuum states at exact resonance.

For excitation into a continuum the resonant denominator no longer picks out a single vibrational-rotational state transition. Instead, all populated vibrationalrotational levels of the ground electronic state are at resonance with some continuum state. The re-emission observed in a given overtone now, rather than resulting from a single vibrational-rotational transition, is composed of a summation of lines resulting from Raman transitions from all the populated levels. This large number of superimposed bands has an averaging effect on the overtone intensities and frequency dependence. An additional difference exists between the discrete and continuum resonance cases. In the discrete case a given line in the re-emission spectrum results from single excited state energy level. Because of the $\Delta J= \pm 1$ dipole transition selection rule, the next closest intermediate state which could contribute to the intensity of that line is one separated by the excited state vibrational spacing. Because of the resonance denominator this "off-resonance" scattering does not contribute significantly to the intensity of the line in question. In contrast, though, in the continuum region the spacing of vibrational levels is effectively infinitesimal, and a given re-emission line gets intensity not only from the continuum state it is in exact resonance with but also from other nearby continuum states that are only slightly off resonance. This serves as an additional averaging effect on the intensity. In the continuum resonance case the various overtones, rather than varying in a haphazard manner as in the discrete case, vary in a systematic manner because of those two averaging effects. Similarly, when the excitation frequency is changed the form of the spectra change in a very continuous and systematic manner as certain vibrational-rotational states become weighted more or less heavily by the combination of the FranckCondon factors and the resonance denominator.

\section{Conclusions from theory}

The behavior of the scattering as the incident laser frequency is varied from nonresonance, to resonance with discrete states, then at higher energies to resonance with continuum states should now be apparent and the differences in the properties listed in Table I reconcilable. In particular, according to Eq. (10) for nonresonance Raman scattering overtones would be expected to be very weak in comparison to the fundamentals. In addition, the spectrum of the fundamentals should be highly structured owing to all the allowed Raman transitions from all the populated vibrationalrotational levels. In contrast, in the continuum region the scattering according to Eq. (11) should consist of numerous overtones of similar intensities and each having a great deal of structure. The scattering intensity in this case is governed by the weighted sums of the Franck-Condon overlap amplutides. When the incident frequency lies in the discrete region, the continuum contribution is now obscured by the strong resonances with specific intermediate states. Again there should be numerous overtones but the intensities of the various overtones should vary erratically because of the variations in the $\langle f \mid i\rangle$ overlap factor in Eq. (12).

When the incident frequency is varied in normal Raman scattering, because it is far from resonance and the resonance denominator may be considered to be constant [see Eq. (9)], all of the frequency dependence is left in the $\omega_{s}^{4}$ factor of Eq. (1). On the other hand, for discrete resonance Raman scattering a small change in incident frequency causes the resonance to occur with a different intermediate state [Eq. (12)] so a completely different spectrum is observed. In continuum resonance Raman scattering the spectrum also changes with excitation frequency, but owing to the averaging effects discussed the changes are smooth and continuous. The Stokes-anti-Stokes ratio for nonresonance scattering is proportional to the population of the state involved. In both discrete and continuous resonance Raman scattering this ratio is not solely population dependent because the overlap factors also affect the experimental ratio.

An upper limit for the scattering time of a Raman process is approximately $\left(\Delta \omega^{2}+\Gamma^{2}\right)^{-1 / 2}$, where $\Gamma$ is the natural linewidth. In the discrete region for exactly resonant excitation, $\Delta \omega=0$, so the scattering time is given by the natural lifetime of the state involved, typically $10^{-5}-10^{-8} \mathrm{sec}$. For incident frequencies off resonance with the discrete transition and for normal off-resonance Raman scattering, the upper limit of the scattering time is given by $1 / \Delta \omega$ (typically $10^{-14}-10^{-15}$ sec in the normal off-resonance Raman scattering case); and in continuum resonance Raman scattering an upper limit may be given by the time needed for the atoms to fly apart $\left(\sim 10^{-12} \mathrm{sec}\right)$. Although the definition of the scattering lifetime becomes unclear in these situations because a direct measurement by time decay can no longer be made, the manifestations of the shortened time for the molecule-photon interaction do have a predictable effect on other physically measurable properties such as foreign gas quenching; namely, quenching is observed only when the gas collision rate is comparable to the time for re-emission. Consequently, for off-resonance Raman scattering and for continuum resonance Raman scattering where the scattering time is fast, quenching of the Raman intensity is observed only at very high foreign gas pressures. On the other hand, for discrete resonance Raman scattering at exact resonance quenching is observed at 
very low foreign gas pressures (fractions of an atmosphere). ${ }^{21}$ As the incident frequency is moved away from exact resonance in the discrete region, the time for re-emission becomes faster and quenching is only observed at higher pressures ( 1 atm).

The depolarization ratio of $S$ and $O$ rotational branches is $\frac{3}{4}$ independent of the incident frequency, but the depolarization factor of the $Q$ branch varies with the excitation frequency. For normal Raman scattering it is typically $0.001-0.01$ and depends of the anisotropy of the polarizability. ${ }^{22}$ In resonance the depolarization behavior has been treated recently by Silverstein ${ }^{10}$ and by Jacon and Van Labeke. ${ }^{11}$ In each case they used perturbation theory and showed that, for high $J$, the discrete resonance Raman $Q$ branch depolarization ratio is $\frac{3}{4}$ and for continuum resonance Raman scattering it is $\frac{1}{8}$ if the angular momentum along the internuclear axis is zero.

\section{EXPERIMENTAL RESULTS AND DISCUSSION}

Spectral and lifetime measurements were made on molecular iodine to test the scattering theories. The well-established electronic states of $\mathrm{I}_{2}$ make it a very suitable choice for such studies. As shown in Fig.2, the excited state potential function $B\left({ }^{3} \Pi_{0}+_{u}\right)$ has a dissociation limit $20162 \mathrm{~cm}^{-1}$ above the $X\left({ }^{1} \Sigma_{0^{+}}\right)$ground state. As such, there are several argon ion laser lines with frequencies above the dissociation limit and several argon ion and krypton ion laser lines below the limit, making the experimental studies in both regions straightforward. Numerous scattering ${ }^{23,24}$ (or fluorescence) studies have been carried out in both regions but in general the emphasis. has not been on detailed frequency dependence studies. We have, under high resolution conditions, studied the spectral changes occurring with several different excitation frequencies above the dissociation limit ${ }^{20}$; and we have measured the spectral and temporal changes ${ }^{25}$ as the incident laser frequency was tuned away from resonance with a transition to a single discrete state. Our results are consistent with the preceding theoretical discussion and with the characteristics listed in the second and third columns of Table I.

The spectral measurements were obtained in a standard manner. Coherent Radiation Models 52 and 53 argon ion lasers were used as the excitation sources, and the light was focused into the gas eous reagent grade $I_{2}$ with a short focal length lens. The scattered light was gathered at right angles and focused onto the slits of a Spex 1401 Double Monochromator. The dis persed light was detected with an ITT FW-130 photomultiplier, amplified, and displayed on a strip chart recorder.

\section{A. Discrete resonance Raman scattering}

In these experiments the reagent grade $\mathrm{I}_{2}$ was distilled several times prior to distillation into a sealed quartz spectroscopic cell. The pressure of the $I_{2}$ in this cell was controlled by placing a low temperature bath around a side arm on the cell. Typically, however, experiments were run with the side arm either at room temperature giving an $I_{2}$ pressure of $\sim 0.25$ Torr or at ice temperature giving a pressure of $\sim 0.03$ Torr. In these experiments great care was taken to insure that no residual air remained in the sample cell, as it would yield spurious data due to quenching.

\section{Frequency spectrum}

The re-emission from $I_{2}$ in the region of discrete transitions is characterized by a series of overtones of erratically varying intensity as shown in Fig. 5 . This spectrum was obtained by single mode excitation in the $5145 \AA$ region. The vibrational fundamental appears at about $213 \mathrm{~cm}^{-1}$ and each overtone is separated by about this frequency. We have arbitrarily stopped the spectrum after the $\Delta v^{\prime \prime}=9$ line, but several more overtones may be readily seen. Each of the overtones results from a transition to a specific intermediate excited $v^{\prime}$ state, in this case $v^{\prime}=58$ of the $B$ state, and then a re-emission to the ground state with the overall change in the vibrational quantum number indicated in the figure. When the rotational selection rule for a dipole transition is $\Delta J= \pm 1$, as it is in $\mathrm{I}_{2}$, a pattern of doublets is observed in the emission, as seen here. This occurs because after the initial transition to the excited state with rotational quantum number $J^{\prime}$ re-emission to final states with $J^{\prime \prime}=J^{\prime}+1$ or $J^{\prime \prime}=J^{\prime}-1$ are possible, resulting in a doublet. The overall change in the ground state vibrational quantum number $J^{\prime \prime}$ then will be 0 and +2 if the initial state is $J^{\prime}-1$ (designated an $R$ transition) and it will be 0 and -2 if the initial state is $J^{\prime}+1$ (designated a $P$ transi-

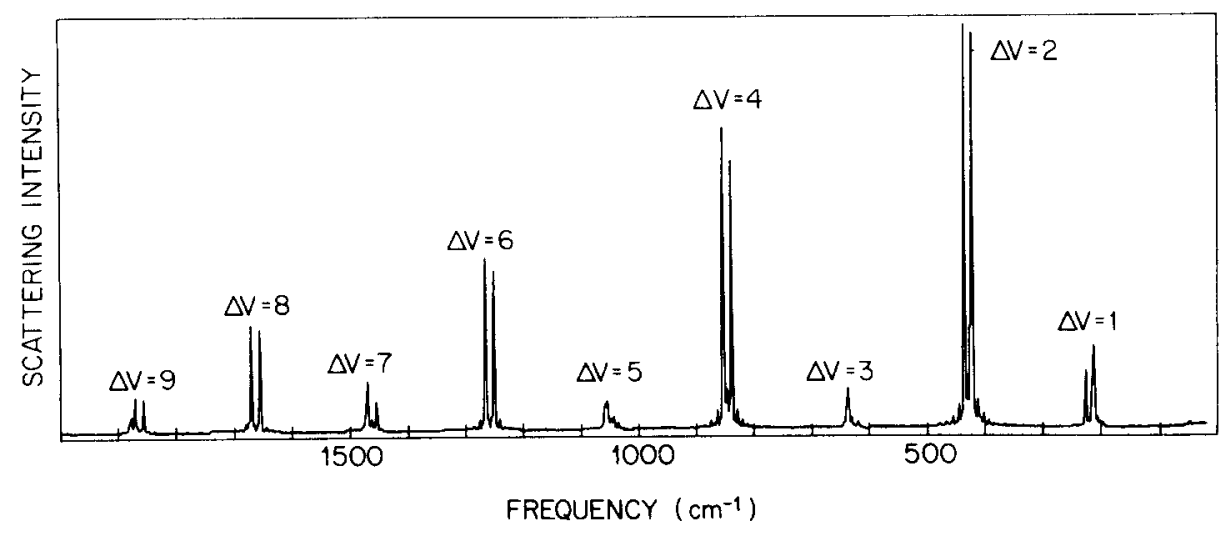

FIG. 5. Survey spectrum of discrete resonance Raman scattering from $\mathrm{I}_{2}$. The laser was set at $5145 \AA$ and single moded with an etalon. 


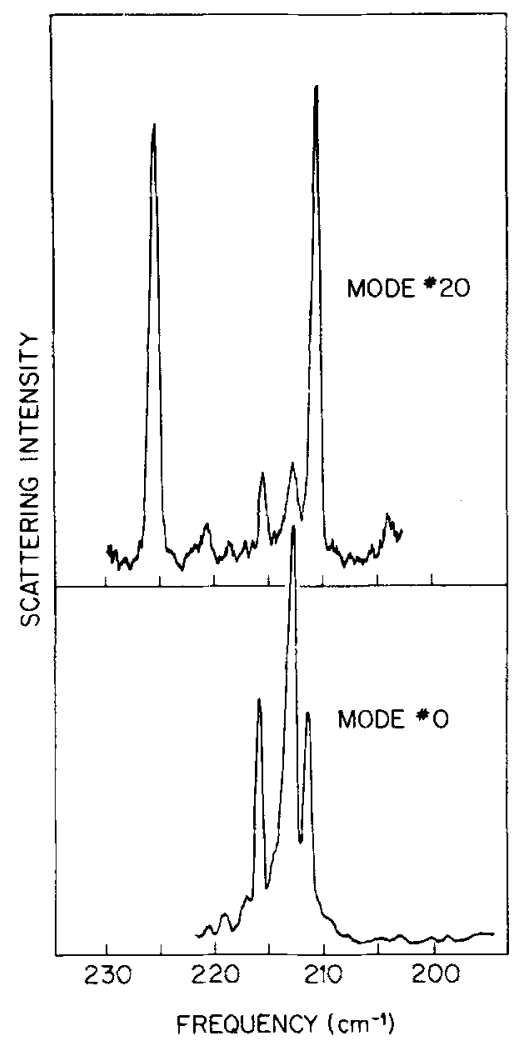

FIG. 6. Re-emission spectra of the vibrational fundamental region of $\mathrm{I}_{2}$ excited in the region of discrete resonance Raman scattering with $5145 \AA$. The laser mode spacing is about 130 MHz. The top spectrum, labeled Mode \#20, was taken at the same laser frequency as the spectrum in Fig。 5 .

tion). The specific re-emission seen in Fig. 5 results from an initial transition from the $v^{\prime \prime}=1$ vibrational and $J^{\prime \prime}=99$ rotational level of the $X$ state to the $v^{\prime}=58$, $J^{\prime}=100$ level of the $B$ state and is therefore termed a $58^{\prime}-1 " ' R(99)$ transition. $^{26}$

As discussed in conjunction with Eq. (12), for discrete resonance Raman scattering only one intermediate state dominates the scattering, resulting in the re-emission doublets seen here. The intensity variation between overtones results from variation in the Franck-Condon overlap factors between this intermediate state and the final state, i. e., $\langle f \mid i\rangle$. The additional structure seen near some of the overtones results from collisional transfer lines and re-emission lines from additional intermediate states that are only slightly out of resonance. In the spectra reported here the linewidth of the individual lines is limited by the spectrometer resolution, but in line shape measurements with a Fabry-Perot spectrometer the expected Doppler plus hyperfine widths have been observed. 27

By changing laser modes it is possible to change from resonance with one intermediate state transition to resonance with another. In Fig. 6 a higher resolution spectrum of the vibration fundamental region of Fig. 5 is shown (labeled Mode \# 20) and compared to a spectrum obtained with a laser mode shifted by $\sim 2.5$ $\mathrm{GHz}$ (labeled Mode \# 0 ). In shifting the frequency by only $2.5 \mathrm{GHz}$, the emission doublet has disappeared entirely and a triplet is now observed. The triplet is seen because the laser is now at resonance with two transitions-one a $P\left[43^{\prime}-0^{\prime \prime} P(13)\right]$ and one an $R\left[43^{\prime}-\right.$ $\left.0^{\prime \prime} R(15)\right]$ resulting in overlapping $Q$ branch $\Delta J^{\prime \prime}=0$ and $O$ branch $\left(\Delta J^{\prime \prime}=-2\right)$ and $Q$ branch and $S\left(\Delta J^{\prime \prime}=+2\right)$ branch contributions, respectively.

\section{Re-emission lifetime}

The lifetime measurements were made on an apparatus built and described ${ }^{28}$ by $\mathrm{R}$. Z. Bachrach in which the time decay is measured by a delayed coincidence technique. Again, Coherent Radiation Model 52 and 53 argon ion lasers were used as the excitation sources. The laser beam was modulated acoustooptically to give rectangular pulses with a $3 \mathrm{nsec}$ rise time although the time response of the electronics used in these experiments was not that fast. The width of the pulse could be varied continuously from about $20 \mathrm{nsec}$ to several $\mu$ secs, but most measurements were made with 100 nsec pulses. The scattered light, after dispersion by the Spex monochromator, was detected by the FW-130 photomultiplier. After feeding the signal to a time-toamplitude converter, a decay curve was then obtained on a pulse height analyzer. Lifetimes ranging from about a $10 \mathrm{nsec}$ lower limit could readily be measured. All the discrete resonance experiments reported here were carried out in the $5145 \AA$ region, where several $I_{2}$ absorption lines may be brought into resonance by tuning the single mode laser through the Doppler profile of the laser line. The laser tunes with discrete mode jumps of $\sim 95 \mathrm{MHz}$ for the Model 53 laser, of $\sim 130 \mathrm{MHz}$ for the Model 52 laser. A total frequency range of nearly $10 \mathrm{GHz}$ is available from this mode selection. In the lifetime measurements the spectrometer resolution was maintained at $2 \mathrm{~cm}^{-1}$ or better so that a single re-emission line could be isolated. All data were obtained in the frequency region of the vibrational fundamental (a frequency shift from the incident laser of about $\left.213 \mathrm{~cm}^{-1}\right)$.

To study the behavior of the lifetime on and off resonance the spectrometer was set to $\sim 216 \mathrm{~cm}^{-1}$ to isolate the $S$ branch of the triplet shown in the bottom of Fig. 6. The modes of the incident laser were then changed so as to at first be on resonance with the $R(15)$ transition resulting in this re-emission and then to gradually move out of resonance with the transition. Although the laser was being shifted away from resonance, because the spectrometer was set at a specific frequency the behavior of a single discrete intermediate state was still being studied without interference from other transitions. Similar experiments at $\sim 213 \mathrm{~cm}^{-1}$ were done on the $Q$ branch [resulting from both the $R(15)$ and $P(13)$ transitions]. The time decay results ${ }^{25}$ of the $S$ branch are shown in Fig. 7. The laser pulse in these measurements was $100 \mathrm{nsec}$ wide. The $0.0 \mathrm{GHz}$ spectrum was obtained by adjusting the laser frequency to obtain the maximum intensity of the re-emission from the $P(13)$ and $R(15)$ transitions, $i_{0} e_{\text {. }}$, to be precisely at resonance. The other time decay measurements were made by shifting the laser modes away from this resonance position. The $1 / e$ lifetime $(\sim 300 \mathrm{nsec})$ of the on resonance $(0.0 \mathrm{GHz}$ in Fig. 7$)$ is significantly 


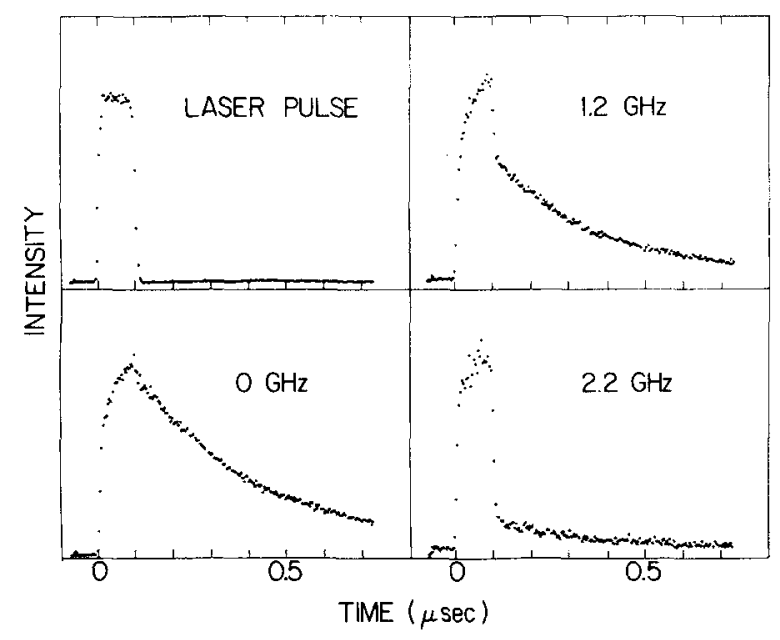

FIG. 7. Time decay data for pulsed excitation of molecular iodine. The spectrometer was set to observe the re-emission from the Raman shifted $S$ branch $\left(\sim 216 \mathrm{~cm}^{-1}\right.$ shift $)$ of the $43^{\prime}-0^{\prime \prime} R(15)$ transition. The laser pulse is shown in the upper left. In the lower left is the time decay data obtained for resonant excitation and on the right are data for off-resonant excitation shifted by $1.2 \mathrm{GHz}$ and $2.2 \mathrm{GHz}$ from exact resonance. The accumulation time was substantially different for each plot ranging from a few minutes for the $0 \mathrm{GHz}$ decay plot to several hours for the $2.2 \mathrm{GHz}$ decay plot.

shorter than that obtained in prior measurements. ${ }^{29}$ This results from diffusion of iodine molecules out of the narrow illuminated region focused on the spectrometer entrance slit, thereby selectively discriminating against the long-lived events. With larger slits, we recorded lifetimes of $\sim 1 \mu \mathrm{sec}$, consistent with prior measurements.

At $1.2 \mathrm{GHz}$ off resonance there are clearly two different lifetime contributions - one short-lived re-emission, decaying in a time limited by our 10 nsec temporal resolution, and one long-lived component with the same decay constant as the $0.0 \mathrm{GHz}$ measurement. Naively, on the basis of a single physical process one may have expected to see a continuous change in lifetime in this region and therefore may be tempted to interpret these results at $1.2 \mathrm{GHz}$ as supportive evidence for two independent physical processes (longlived resonance fluorescence and short-lived Raman scattering). However, consideration of the magnitudes of the quantities involved shows that it results from the complicated line shape of the iodine absorption. It must be remembered that a frequency shift as small as $20 \mathrm{MHz}$ from resonance results in an uncertaintylimited lifetime of $10 \mathrm{nsec}$, the lower limit of our temporal resolution. In addition, the absorption lineshape of iodine is not determined by its natural (radiative and nonradiative) lifetime, but primarily by the Doppler broadening and the hyperfine splitting, resulting in a total linewidth of over $1 \mathrm{GHz}$, Consequently, incident frequencies in the wings of the absorption tail (see 1.2 GHz spectrum in Fig. 7) are on resonance $(\Delta \omega<100$ $\mathrm{kHz}$ ) with some components within the Doppler profile, and very far off resonance $(\Delta \omega>20 \mathrm{MHz})$ with others, giving the long- (natural lifetime) and short- (<10 nsec) lived contributions, respectively.
Further off resonance at $2.2 \mathrm{GHz}$ in Fig. 7, the reemission is dominated by the fast response and only a weak residual slow response remains. Decay curves similar to the $2.2 \mathrm{GHz}$ curve were obtained from 1.6 $\mathrm{GHz}$ off resonance to $2.6 \mathrm{GHz}$. Because of the weakness of the re-emission from this $R(15)$ transition at $2.6 \mathrm{GHz}$ off resonance, measurements beyond this point were not made. In measurements between 0.0 and $\sim 1.5 \mathrm{GHz}$ off resonance the excitation frequency was still in the wings of the Doppler tail, so as the incident frequency was moved away from resonance the intensity of the "long"-lived component progressively became weaker with respect to the "short"-lived one. However, for incident frequencies beyond $1.6 \mathrm{GHz}$ off resonance where the incident frequency was in the Lorentzian wings of the absorption the relative intensities of the two components no longer changed.

It is worthwhile to note that the on-resonance $(0 \mathrm{GHz}$ in Fig. 7) predicted time response for $\gamma \gg \Gamma$ given by Eq. (21) and depicted in Fig. 4 is not consistent with the response actually observed. Specifically, the leading edge of the pulse is predicted to have a rise quadratic in $\left(1-e^{-\Gamma^{t}}\right)$, which is very different from the dependence observed in Fig. 7. This difficulty results from two effects. First, in the decay spectrum of Fig. 7, the peak power of the laser was $\sim 50 \mathrm{~mW}$, causing the resonant transition to saturate. In Fig. 8 we present on-resonance time decay data obtained with $\sim 0.1 \mathrm{~mW}$ of power and an $\mathrm{I}_{2}$ pressure of 0.25 Torr. Note in particular the difference in the leading

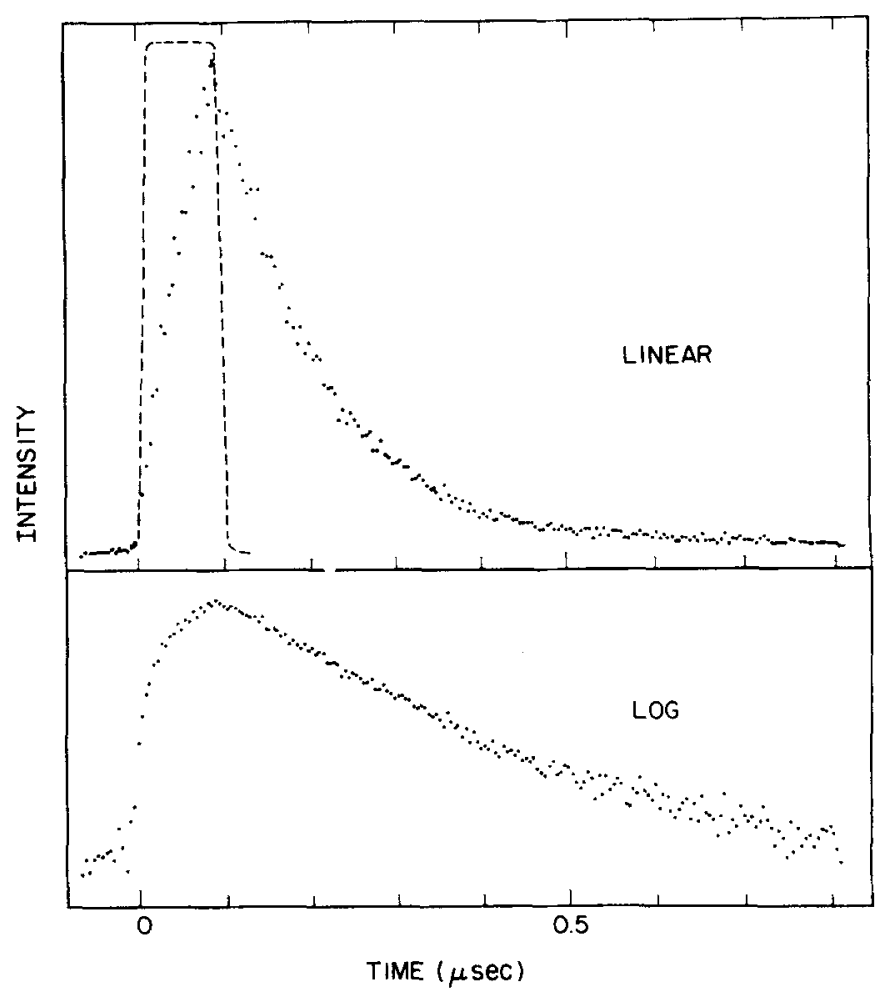

FIG. 8. Linear and logarithmic display of time response resulting from excitation of room temperature $\mathrm{I}_{2}$ at exact resonance. To obtain these data the laser power was lowered to $0.1 \mathrm{~mW}$ to reduce saturation effects. The dashed line corresponds to the position of the excitation pulse. 
edge of the pulse. In this figure we believe the deviation from linearity in the decay portion of the $\log$ plot results from photomultiplier noise. A second effect causing the pulse to deviate from the simple $\left(1-e^{-\Gamma t}\right)^{2}$ dependence predicted by Eq. (21) results from the Doppler broadening. This broadening is typically much larger than a natural linewidth for these transitions, so that for excitation frequencies near the center of the Doppler broadened profile, the re-emission intensity from the ensemble is to an excellent approximation given by $\int_{-\infty}^{\infty}|G(t)|^{2} d \Delta \omega$. This integral is easily carried out using $\mathrm{Eq}$. (20), with the following result:

$$
\begin{aligned}
& \int_{-\infty}^{\infty}|G(t)|^{2} d \Delta \omega=\frac{\pi}{(\gamma-\Gamma)(\gamma-2 \Gamma)}\left\{\left[\frac{\gamma^{2}}{\Gamma}\left(1-e^{-2 \Gamma t}\right)-2 \gamma\left(1-e^{-\gamma t}\right)\right]-(\gamma-2 \Gamma)\left(1-e^{-\gamma t}\right)^{2}\right\} \\
& =\frac{\pi}{(\gamma-\Gamma)(\gamma-2 \Gamma)}\left\{\left[\frac{\gamma^{2}}{\Gamma}\left(1-e^{-2 \Gamma T}\right)-2 \gamma\left(1-e^{-\gamma T}\right)\right] e^{-2 \Gamma(t-T)}-(\gamma-2 \Gamma)\left(1-e^{-\gamma T}\right)^{2} e^{-2 \gamma(t-T)}\right\} \quad t \geq T . \\
& 0 \leq t \leq T .
\end{aligned}
$$

In Fig. 9 this function is plotted, and in the insert in the upper right it is compared with the Fig. 8 data for the first $100 \mathrm{nsec}$. In the calculation $(2 \Gamma)^{-1}$ was taken to be 125 nsec, the measured value in Fig。 8 , and $(2 \gamma)^{-1}$ was taken to be 3 nsec. The agreement between the observed and calculated spectra is quite satisfactory.

In the direct lifetime measurements reported here, we conclude that on resonance the lifetime is slow and off resonance it is fast. As discussed, the uncertainty principle allows direct measurement only of an upper limit to the off-resonance lifetime. However, we might expect quenching behavior to serve as an indirect measure of intermediate lifetimes. By adding a foreign gas to the $I_{2}$, quenching of the re-emission intensity would

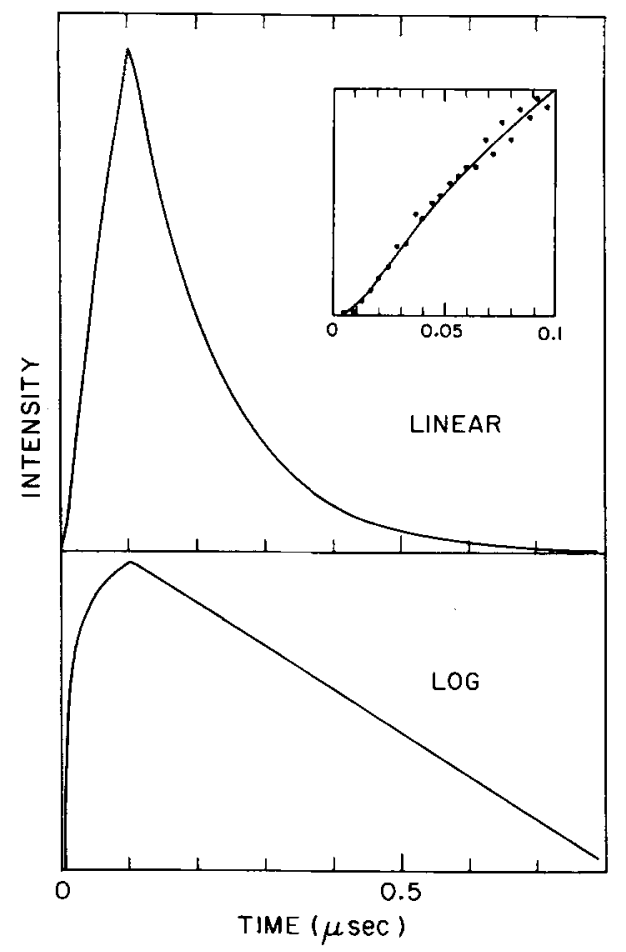

FIG. 9. Linear and logarithmic display of time response predicted from Eq. (24) with a 100 nsec pusle. $(2 \gamma)^{-1}$ was taken to be $3 \mathrm{nsec}$ for the laser pulse rise time and $(2 \Gamma)^{-1}$ was taken to $125 \mathrm{nsec}$ for the room temperature $\mathrm{I}_{2}$ response time. The insert is a linear display of the first $100 \mathrm{nsec}$ of the response, and for comparison data points taken from Fig. 8 are shown. not be expected until the collision rate equals the reemission lifetime. In Fig. 10 we present quenching data at several different excitation frequencies obtained by adding foreign gas, $\mathrm{N}_{2}$, to the iodine cell. In Fig. 10 the labels on each curve correspond to the frequency shift in laser modes (one mode $\simeq 130 \mathrm{MHz}$ ) with the $P(13)$ and $R(15)$ transitions. The spectrometer was set to observe the fundamental Raman re-emission at a frequency shift of about $216 \mathrm{~cm}^{-1}$. As expected, when the incident frequency is near the center of the resonance the foreign gas very rapidly quenches the fluorescence. Off resonance considerable foreign gas had to be added to the cell before a change in the fluorescence intensity was seen. One would like to conclude from this that a lifetime for re-emission could be calculated from that pressure. However, that would only be possible if all of the broadening resulted from inelastic collisions, i. e., those lifetime broadening col-

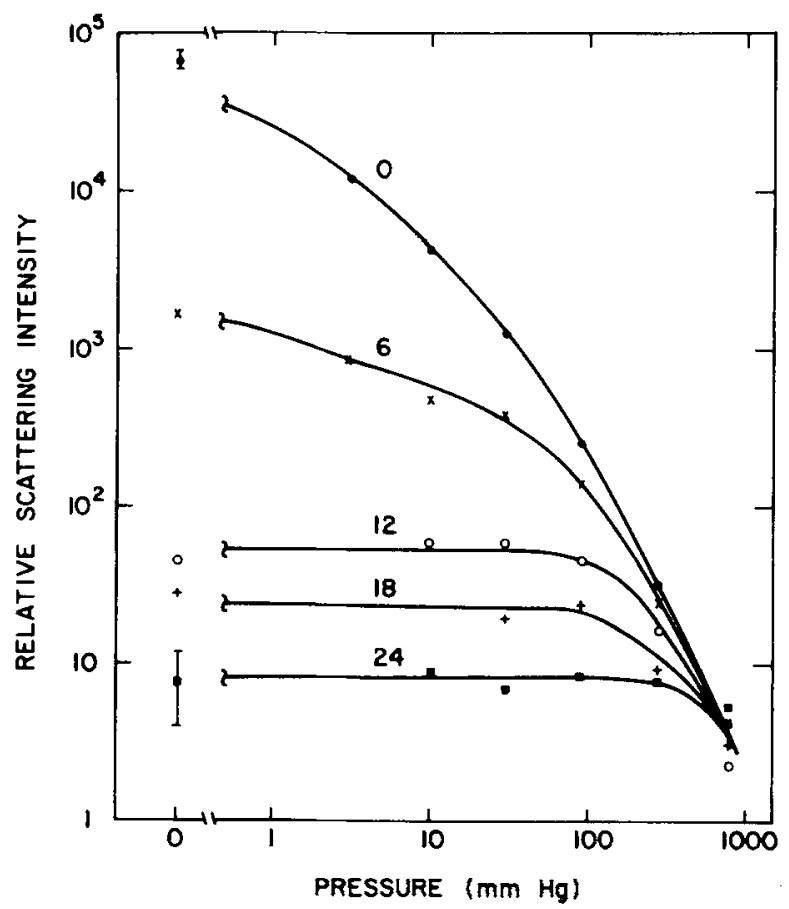

FIG. 10. Quenching behavior of the $I_{2}$ re-emission upon the addition of $\mathrm{N}_{2}$ gas. The curves are labeled by laser modes shifted from exact resonance with the $P(13)$ and $R(15)$ lines. The laser mode spacing is $130 \mathrm{MHz}$. 


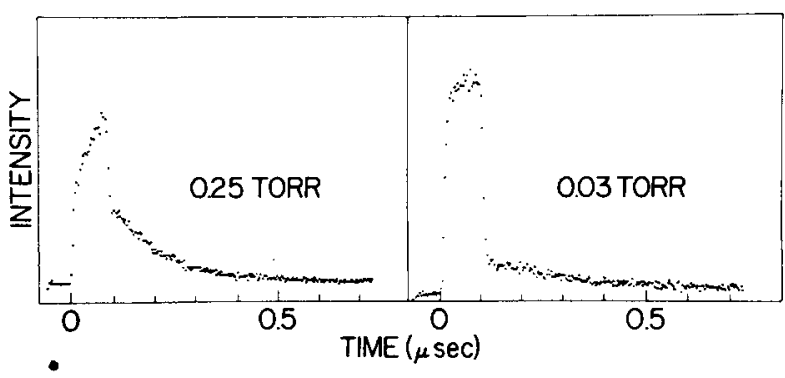

FIG. 11. Time decay data for the $I_{2}$ re-emission obtained at two different $I_{2}$ pressures.

lisions in which the molecule is knocked into a completely different quantum state. Pseudoelastic collisions, though, which interrupt the phase but leave the molecule in the same quantum state, may also take place. Broadening from such collisions in an offresonance experiment effectively increases the absorption linearly with pressure, but the quenching decreases the fluorescence linearly with pressure, giving a pressure independent intensity in the low pressure region. Finally a point is reached where the absorption saturates so the overall fluorescent intensity starts to decrease. The pseudoelastic collisions then, as originally pointed out by St. Peters and Silverstein, ${ }^{30}$ give a behavior qualitatively the same as one would expect from a lifetime broadening mechanism, and conclusions may not be drawn about the system lifetime.

\section{Collision effects}

To study the origin of the long-lived remnant shown in Fig. 7 at $2.2 \mathrm{GHz}$, we have made a determination of the effect increased pressure has on the lifetime response. ${ }^{19}$ The decay curves obtained in Fig. 7 were obtained at $\sim 0.03$ Torr of $I_{2}$. In Fig. 11 the decay curves obtained at $\sim 1.7 \mathrm{GHz}$ off resonance with $\mathrm{I}_{2}$ pressures of 0.25 Torr and 0.03 Torr are presented. At 0.25 Torr of $\mathrm{I}_{2}$ there are two prominent components to the scattering. One has a fast rise and decay (it follows the excitation pulse) and therefore results from the Raman contribution to the scattering [first term in braces in Eq. (23)]. The other contribution has an exponential rise and decay and we attribute it to the redistribution term. It has a lifetime of about $125 \mathrm{nsec}$, substantially shorter than the natural lifetime, as expected, since at these pressures the lifetime is given by $1 /\left(\gamma_{N}+\gamma_{I}\right)$.

As the $I_{2}$ pressure is lowered, $\gamma_{E}$ decreases, so the relative intensity of the redistribution term to that of the Raman term also decreases. Consequently, as seen in Fig. 11, at 0.03 Torr of $I_{2}$ the long-lived contribution is greatly reduced with respect to the shortlived component. The lifetime for the slow component is longer than that of the redistribution term at the higher pressure although not as long as expected on the basis of the natural lifetime. It exhibits a shorter decay constant than expected owing to the spatial resolution imposed by the narrow spectrometer slits used in the experiment.

As a further study of the effects collisions have on the spectral re-emission, high resolution spectra were obtained. The measurements were carried out by directing the scattered light through an interference filter centered at the Stokes frequency shift $\left(\sim 213 \mathrm{~cm}^{-1}\right)$ and into a piezoelectrically scanned Fabry-Perot interferometer with a $10 \mathrm{GHz}$ free spectral range. The measurements were made in a near forward scattering geometry to reduce the effects of Doppler broadening and, as in the lifetime measurements, the excitation frequency was shifted $1.7 \mathrm{GHz}$ away from the center of the resonant transitions. The resulting spectrum for 0.25 Torr of $I_{2}$ is depicted by the points in Fig. 12 . The sharp features in the spectrum result from Raman scattering from the $S, Q$, and $O$ branches (in different orders of the interferometer) and the laser line which leaked through the narrow band filter. In addition to the sharp features there is a broader underlying structure. We attribute this to redistribution scattering. The sharp Raman features have widths limited by Fabry-Perot resolution. In contrast, the redistribution scattering width regains the full width of the Doppler broadened transition.

The solid curve in Fig, 12 is a calculation of the spectrum obtained in the following manner. The frequencies of the sharp $S, Q$, and $O$ Raman lines were fitted to coincide with the experimental frequencies and were assigned the expected 1, 2, 1 relative intensity ratios. Shifted $1.7 \mathrm{GHz}$ away from each of the sharp Raman lines a redistribution band is located. Its shape

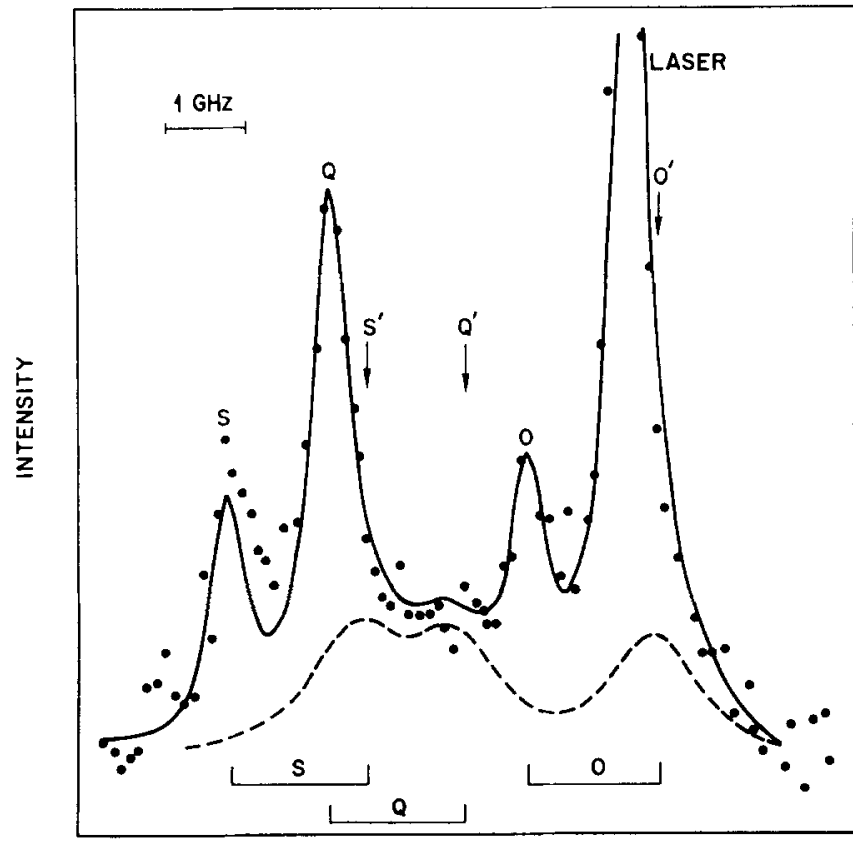

FREQUENCY

FIG. 12. High-resolution frequency spectrum of $214 \mathrm{~cm}^{-1}$ Stokes re-emission of $\mathrm{I}_{2}$ obtained with $1.7 \mathrm{GHz}$ off-resonance excitation. $S^{\prime}, Q^{\prime}$, and $O^{\prime}$ correspond to the positions of the rotational branches obtained with resonant excitation. The points are the experimental data. The solid line is a theoretical curve, and the dashed line is the redistribution-scattering contribution. The bars at the bottom indicate the 1.7 $\mathrm{GHz}$ shift between each Raman-redistribution pair. 
was determined from an optical absorption spectrum we obtained and its intensity corrected for an accidental absorption at the $Q$ branch resonance frequency. The resulting spectrum was then folded into a Lorentzian interferometer response function and a best fit to the data was obtained by adjusting the relative intensity of the total Raman to the total redistribution contributions. The resulting redistribution intensity after convolution with the slit function is depicted by the dashed curve in Fig. 12. For the spectra reported in Fig. 12 we found that the redistribution term had an integrated intensity of about 0.8 that of the Raman contribution. We believe the minor remaining discrepancies between the observed and calculated spectra result primarily from additional low intensity bands resulting from inelastic collisional transfer processes.

The possible contribution from inelastic collisions merits more discussion. Experiments were carried out to determine if such collisions significantly influence the time decay and frequency data. First the spectral structure of the fundamental vibrational transition region $\left(\sim 180 \mathrm{~cm}^{-1}-250 \mathrm{~cm}^{-1}\right.$ shifted from the laser line) was examined in detail. As may be seen in Fig. 6 , where part of this spectrum is shown, transfer bands from inelastic collisions make only weak contributions to the spectrum. If substantial transfer band intensity from inelastic collisions were present in the $1 \mathrm{~cm}^{-1}$ frequency window of the $S$ branch used to measure the time decay, then near the region of the triplet strong transfer lines would be observed. Indeed, such lines are observed when several millimeters of foreign gas are added to the iodine. We conclude from their absence in our experiments that inelastic collisional transfer processes do not make a significant contribution to the lifetime data.

Since the relative intensities of the two contributions (instantaneous and long lived) in Fig. 11 for 0.25 Torr pressure are approximately equal and since the broad and sharp features in Fig. 12 are also of the same order, we believe that in the frequency spectrum of Fig. 12 we are in fact observing the same contributions as in the time decay spectrum, Fig, 11, namely a contribution from pseudoelastic collisions. However, in the spectral measurements it was necessary to determine if inelastic transfer lines passed by the narrow band filter and the Fabry-Perot interferometer influenced the observed spectrum. Therefore we measured the total transmission through the narrow band filter with the low resolution $\left(1 \mathrm{~cm}^{-1}\right)$ spectrometer and compared the integrated intensity of the transfer lines to that of the $S, Q, O$ triplet. From this measurement we found that the integrated intensity of the transfer bands from inelastic collisions is a factor of 3 too small to account for the redistribution data of Fig. 12. We therefore conclude that the slow decay in Fig. 11 at 0.25 Torr and the broad features in Fig. 12 result from pseudoelastic collisional redistribution processes.

In the time decay spectra then, we may conclusively assign the long-lived component at 0.25 Torr as resulting from pseudoelastic collisional transfer processes. Although we believe that the long-lived com-

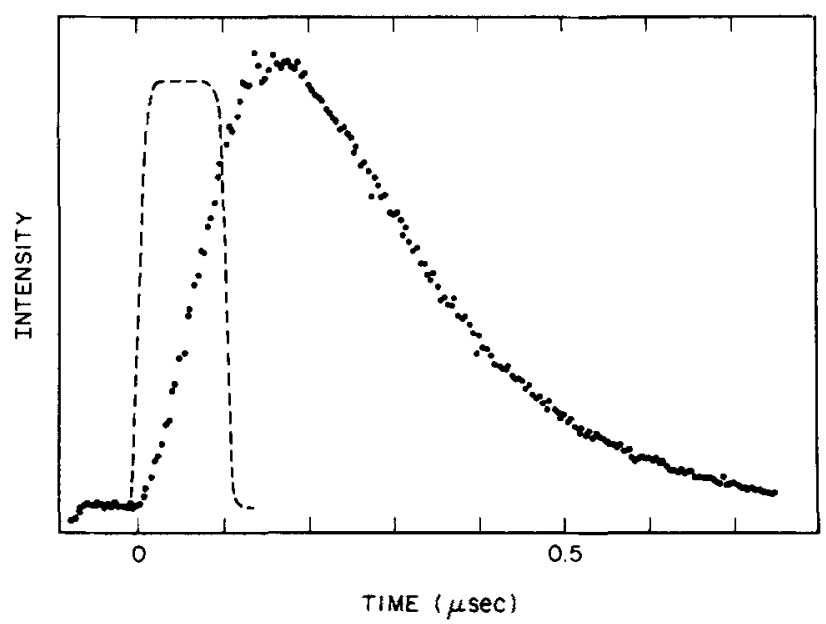

FIG. 13. Time decay spectrum (data points) of a collisional transfer line resulting from inelastic scattering. The width of the laser pulse was $100 \mathrm{nsec}$ and its position is indicated by the dashed line.

ponent in the 0.03 Torr spectrum results from the same origin, we cannot rule out the possibility that it results from excitation at the resonance frequency due to the spectral distribution of the laser pulse. ${ }^{9} \mathrm{Ex}-$ periments done at lower pressure should elucidate this point.

In the off-resonance pressure sensitive lifetime data of Fig. 11 the contribution from redistribution scattering decays exponentially. If this contribution could be isolated in an experiment with exactly resonant excitation, for a $100 \mathrm{nsec}$ pulse used in this work it should have a substantially different behavior, reaching a maximum somewhat after the termination of the excitation pulse due to the time evolution of the prepared state. Because of the limited frequency resolution in our time decay experiments, we have not been able to observe this effect for the pseudoelastic collisional processes. However, we have observed it by studying the time decay of a transfer line resulting from inelastic collisions. As shown in Fig. 13, the time decay curve ${ }^{31}$ of a collisional transfer line reaches its maximum after the excitation pulse is turned off.

\section{B. Continuum resonance Raman scattering}

In experiments ${ }^{20}$ above the dissociation limit in the continuum region the gaseous iodine was in a sample cell held at a temperature of about $420^{\circ} \mathrm{K}$. By setting the temperature of an $\mathrm{I}_{2}$ reservoir to about $360^{\circ} \mathrm{K}$, an $I_{2}$ pressure of about 25 Torr was maintained. Spectra were obtained at excitation wavelengths of 4965,4880 , 4765 , and $4579 \AA$ with a power level of about $500 \mathrm{~mW}$. One half an atmosphere of gaseous nitrogen was introduced to one sample cell in order to measure the iodine temperature during a scattering experiment by monitoring the rotational distribution of the $\mathrm{N}_{2}$. The addition of the $\mathrm{N}_{2}$ had a negligible effect on the structure and the intensity of the re-emission. The iodine temperature due to heating in the laser beam was determined to be $520-620^{\circ} \mathrm{K}$ depending on the excitation frequency. 


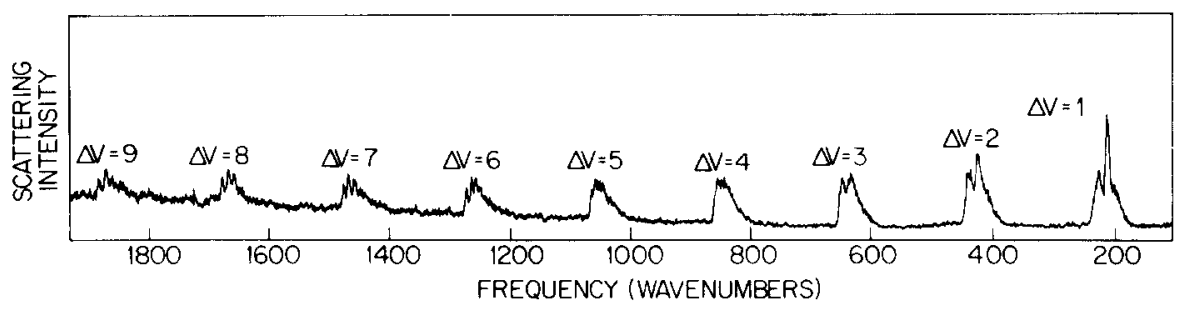

FIG. 14. Survey spectrum of continuum resonance Raman scattering from $\mathrm{I}_{2}$. The laser excitation wavelength was $4880 \AA$.
When the excitation frequency is brought into the continuum region many overtones are again observed, as has been reported previously ${ }^{1,32}$ and as is shown in Fig. 14 for excitation with $4880 \AA$. In contrast to the discrete case, the relative intensity of one overtone to the next varies in a systematic way. The structure within each overtone is real and results from the $O, Q, S$ branches of the multitude of vibrational and rotational transitions that are occurring. This structure may be better seen under higher resolution conditions as shown in Fig. 15 for the overtone corresponding to $\Delta v^{\prime \prime}=5$. As was first pointed out by Kiefer and Bernstein, ${ }^{32}$ consideration of the Fortrat diagram for each of the rotational branches readily explains the origin of the structure in the spectra. In this figure the sharp features are all $S$ branches and their labeling corresponds to the initial and final vibrational levels, respectively, of the Raman transition. Each line consists of several transitions within the $S$-branch manifold. Owing to the energy dispersion of the $Q$ and $O$ transitions as seen in a Fortrat diagram, ${ }^{32}$ a well-defined band head is absent, and therefore these branches are not well-defined discrete features in the spectrum. Instead, they lead to the broad underlying band seen in each spectrum.

In the region of discrete resonance Raman scattering a change in the laser frequency of $1 \mathrm{GHz}$ resulted in a dramatic change in the scattering spectrum。 In Fig. 15, in changing the laser frequency from 4965 to 4579 $\AA$, over $1000 \mathrm{~cm}^{-1}$, the appearance of the spectrum has changed in a very continuous and systematic way. In particular, the relative intensity of the various hot bands has changed from a condition at long wavelengths where low $v^{\prime \prime}$ hot bands dominate to a condition at shorter wavelengths where higher $v^{\prime \prime}$ hot bands dominate. This systematic variation in which higher hot bands dominate at higher laser energies is true of all the overtones. Such a smooth variation in structure with incident frequency is expected on the basis of Eq. (11) and occurs because of the continuous variation in magnitude of the overlap factors as a function of excited state energy.

To more quantitatively interpret these continuum resonance Raman spectra we have numerically calculated $\alpha_{g f}$ in $\mathrm{Eq}$. (11) for depolarized continuum resonance Raman scattering for several overtones and laser frequencies. In this calculation Eq. (11) was evaluated for transitions occurring via the $B$ continuum states only. We have neglected to consider the discrete states of the $B$ potential well and the continuum states of the ${ }^{1} \Pi_{14}$ potential whose shape, indicated schematically in Fig. 16, is not well known. The potential curves for both the $B$ state ${ }^{33,34}$ and the $X$ state st $^{35}$ were obtained by interpolating between turning points which were empirically determined. Since the turning point data only included the banded region of the $B$ states, we extrapolated the small internuclear distance side of the curve with an $a+b / r^{12}$ curve.

Once the potential curves were obtained, continuum wavefunctions for the $B$ state and the bounded wavefunctions for the $X$ state were calculated numerically on a computer. The wavefunctions were calculated by solving a radial Schrodinger equation by an equivalent finite difference equation. ${ }^{36}$ From the resulting wavefunctions, overlap integrals between the bounded vibrational states of the $X$ potential and the continuum states of the $B$ potential were calculated numerically.

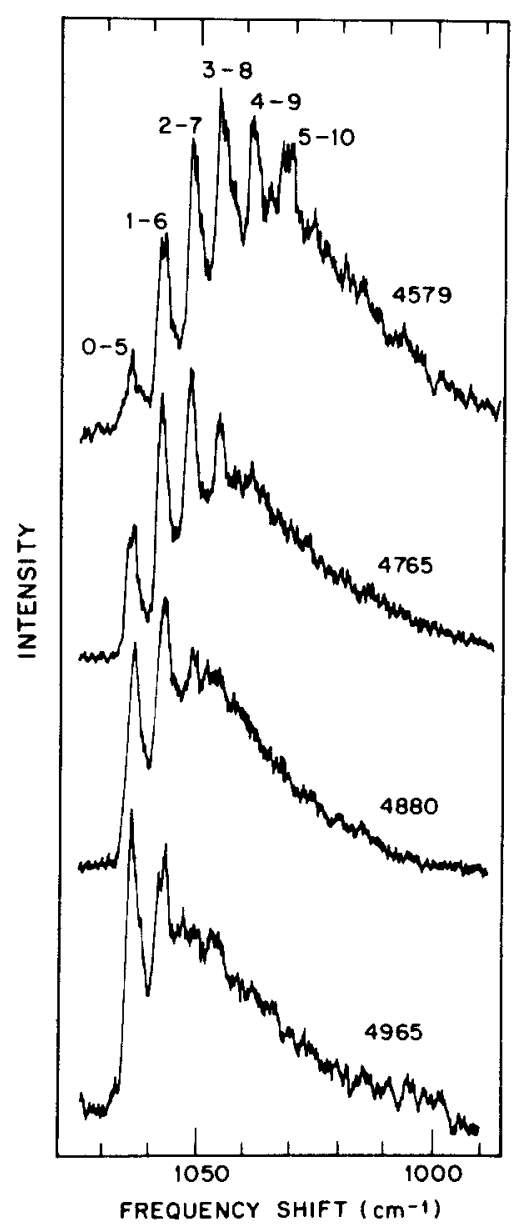

FIG. 15. Structure of the $\Delta v^{\prime \prime}=5$ transition for several laser frequencies in the continuum resonance Raman scattering region. The sharp lines in the spectrum are $S$-branch transitions and the initial and final vibrational quantum numbers for each of these lines are assigned in the upper spectrum. 


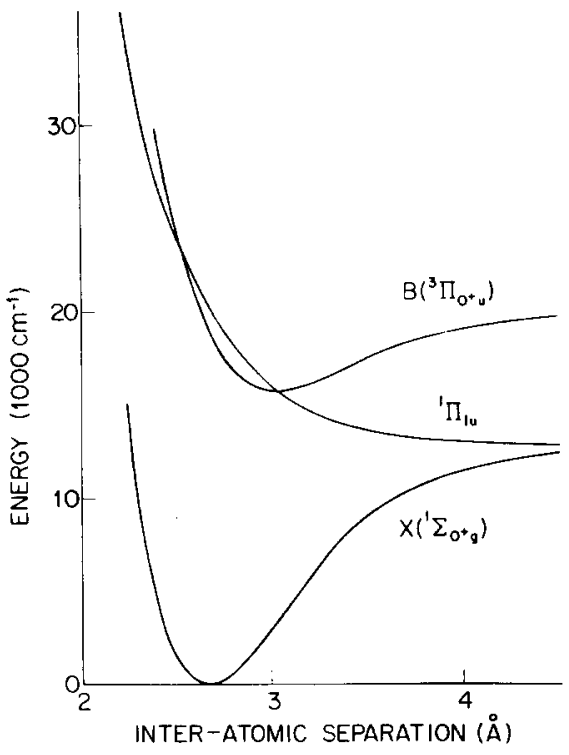

FIG. 16. Potential energy curves of $\mathrm{I}_{2}$.

To evaluate the Raman intensity, overlap factors were calculated between all the relevant ground state vibrational levels and a series of continuum levels. A small enough net of continuum levels was selected so that overlap factors for continuum wavefunctions at energies not calculated could be obtained by interpolation. The potential curves were the $J=0$ curves. At high $J$ the shapes of the potentials change, and they both shift towards larger internuclear separation. ${ }^{37}$ However, the effect of the rotational energy term does not substantially affect the shape of either the bottom of the $X$ potential or the high energy region of the $B$ potential near the inside turning point. Since the dominant contribution to the overlap integrals comes from the region near this turning point, in the calculation reported here we have neglected changes in shape of the potential curves and have accommodated the changes in energies of the potentials as a result of the rotational term, by shifting their relative positions by the appropriate rotational energies. In this manner we have calculated the intensities of Raman transitions originating from the first ten thermally populated vibrational levels of the $X$ state for $J$ values up to $J=400 .^{38}$

Since only one intermediate electronic state is being considered, Eq. (11) may be rewritten to accommodate the singularity as

$$
\begin{aligned}
\alpha_{g f}=\left[M\left(\xi_{0}\right)\right]^{2} \frac{1}{\hbar} & \left\{\rho \int_{0}^{\infty} \frac{\langle f \mid i\rangle\langle i \mid g\rangle \rho\left(\omega_{i}\right)}{\omega_{g i}-\omega_{L}} d \omega_{i}\right. \\
& \left.-\left.i \pi\langle f \mid i\rangle\langle i \mid g\rangle \rho\left(\omega_{i}\right)\right|_{\omega_{i}=\omega_{L}}\right\},
\end{aligned}
$$

where $\rho\left(\omega_{i}\right)$ is the density of continuum states.

Using $\alpha_{g f}$ we may now write Eq. (1) for each transition to include the rotational and vibrational population factors and the $O, Q$, and $S$ brand intensity factors as follows:

$I_{s}=\frac{8 \pi P}{9 c^{4}} I_{L} \omega_{s}^{4} e^{-\hbar \omega_{g} / k T}(2 J+1) b_{J} e^{-B J(J+1) h c / k T}\left|\alpha_{g f}\right|^{2}$.
Here $P$ is a constant that includes the vibrational and rotational partition functions, $B$ is the iodine $X$ state rotational constant, $b_{f}$ is the rotational branch intensity factor as given by Placzek and Teller, ${ }^{39}$ and the zero of energy was taken to be the bottom of the $X$ potential well. Using this expression the Raman intensity for each vibrational rotational transition was calculated. All the rotational levels with significant population (up to $J=400$ ) and hot bands originating from the $v^{\prime \prime}=0$ to $v^{\prime \prime}=9$ ground electronic state levels were included in the calculation for each overtone. The calculated spectra were finally produced by convoluting the spectral intensity with a two wave number rectangular slit function.

In carrying out the calculation we have assumed that $M(\xi)$ is constant. On the basis of calculations ${ }^{33}$ of the electronic transition moment in $\mathrm{I}_{2}$ we would expect this approximation to be valid since $M(\xi)$ is nearly a constant over the internuclear separation range of interest.

The contributions to the total intensity from the real and the imaginary amplitudes in Eq. (25) are oscillatory with laser frequency, as shown in Fig. 17. Here we have plotted the real (solid curve) and the imaginary (dashed curve) transition amplitude as a function of the frequency above the $B$ dissociation limit for the Raman transition $v^{\prime \prime}=4-7$. Note that these amplitudes make approximately equal contributions to the intensity and oscillate in a mutually compatible way-for incident frequencies in which one contribution is small, the other contribution tends to pass through a maximum. Similar behavior was observed for the other transitions.

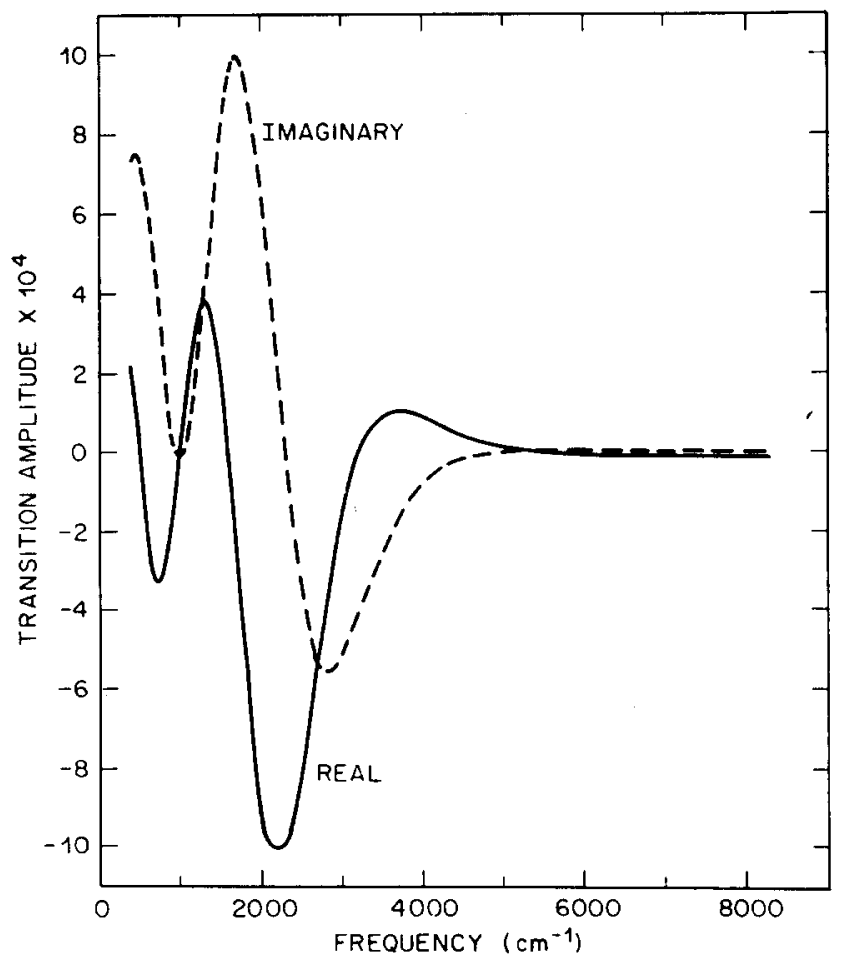

FIG. 17. Variation of the real and imaginary parts of the transition amplitude with incident laser frequency for the Raman transition $v^{\prime \prime}=4 \rightarrow 7$. The solid curve is the real contribution and the dashed curve is the imaginary contribution. 
It should be noted that this oscillatory behavior of the imaginary part of the amplitude has been observed directly $^{40}$ in the discrete-continuum emission spectra in the $\mathrm{I}_{2} E-B$ transition.

In Fig. 18 we show the comparisons of the calculated and observed spectra for the $\Delta v^{\prime \prime}=8$ overtone and in Fig. 19 for the $\Delta v^{\prime \prime}=3$ overtone. The structure in Fig. 18 is similar to the $\Delta v^{\prime \prime}=5$ overtone shown previously. However, in the $\Delta v^{\prime \prime}=3$ spectrum in Fig. 19 for the 4965 and $4880 \AA$ excitations, there is a clear distinction between the $S$ - and $Q$-branch envelopes. This distinction has disappeared in the $4579 \AA$ spectrum. For high energy excitations these separate $S$ and $Q$ envelopes get washed out by the large number of hot bands in the spectrum owing to the tendency of higher hot bands to become more prominent with higher laser frequencies. On the other hand, at lower excitation frequencies where only the transitions originating from low $v^{\prime \prime}$ levels are strong, the $S$ and $Q$ envelopes may be resolved.

The calculated spectra for these two sets of overtones are shown on the right hand side of Figs. 18 and 19. Since the $4965 \AA$ excitation line is less than $100 \mathrm{~cm}^{-1}$ above the dissociation limit, a spectrum for this wavelength was not calculated because contributions to the scattering from the discrete states are expected to be large for this excitation line. The frequencies of the dominant freatures in the calculated spectra agree with those in the observed spectra within experimental er-

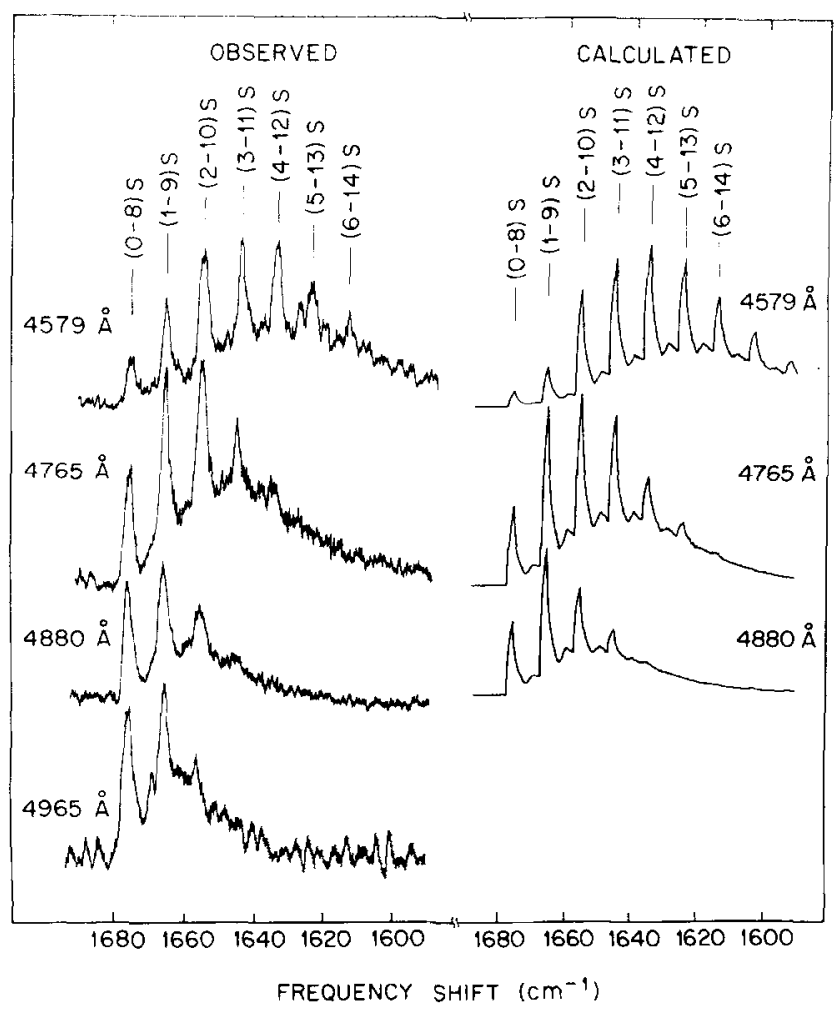

FIG. 18. Experimentally observed and theoretically calculated iodine resonant Raman spectra for the $\Delta v^{\prime \prime}=8$ transition. $S$ numbers refer to the initial- and final vibrational state assignments of the $S$ branches.

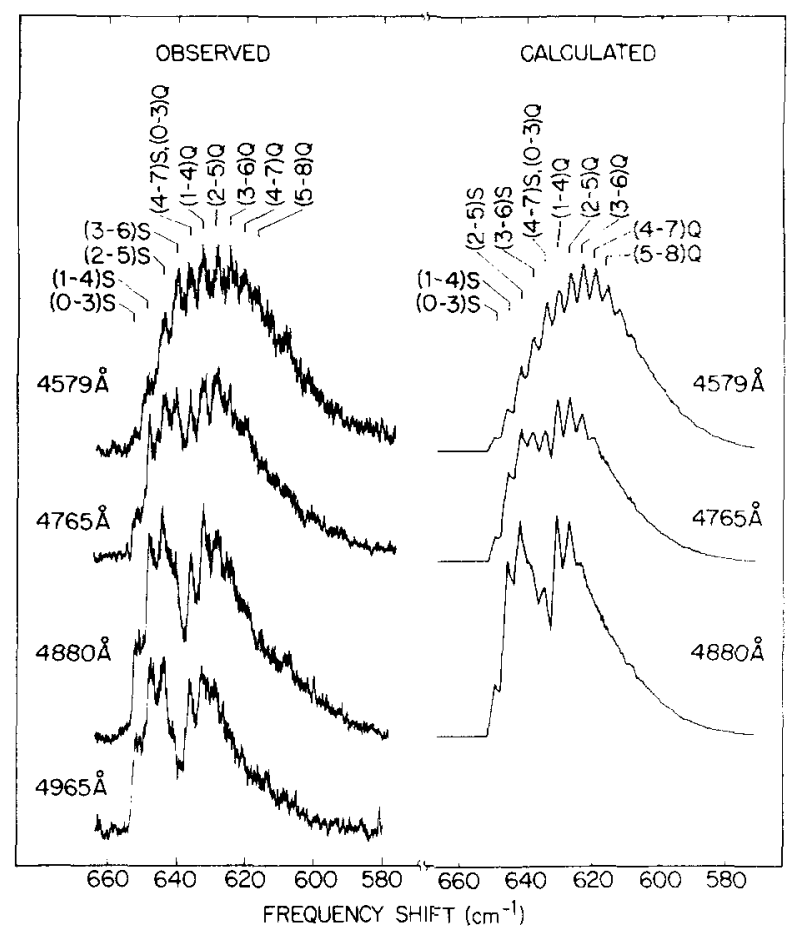

FIG. 19. Experimentally observed and theoretically calculated iodine resonant Raman spectra for the $\Delta v^{\prime \prime}=3$ transition. $S$ and $Q$ numbers refer to the initial- and final-vibrational state assignments of the $S$ and $Q$ branches.

ror. The absolute intensity of the calculated spectrum for each laser line was scaled to the experimental intensity. Except for this scale factor, these spectra were calculated with no adjustable parameters.

In the calculated spectra, as in the observed spectra, the trend toward the dominance of higher hot bands at higher laser frequencies may be seen. This results from changes in the magnitude of the Franck-Condon factors with incident laser frequency. In all the calculated spectra there is a general weakness of transitions originating from the lowest vibrational states (i.e., $v^{\prime \prime}=0,1$ ) as compared with the observed spectra. We believe this discrepancy results from our approximation that the spectral features are completely governed by the $B$ continuum states. We have neglected contributions to the sum in Eq. (11) from the ${ }^{1} \Pi_{1 u}$ repulsive state and from the discrete levels of the $B$ state.

The ratio of the intensity of the second to seventh overtone was measured for each laser excitation frequency, and a calculated ratio was also obtained. For spectra taken at 4579 and $4765 \AA$ the calculated and measured intensity ratios agreed within experimental error $(\sim 10 \%)$. However, at $4880 \AA$ the ratios differed by about $50 \%$. Again we believe this difference at $4880 \AA$ results from including only the $B$ continuum states in our intermediate-state calculation. We have not measured the relative intensities of a given overtone with laser frequency because variations in the absorption coefficient over the range of interest and also the thermal-lens effect complicate the experimental measurement. 
It is expected that the discrete states may make contributions to the scattering intensities especially for laser wavelengths near the dissociation limit. In addition, recent photodissociation ${ }^{41}$ and optical-absorption $^{33}$ experiments have indicated that the ${ }^{1} \Pi_{1 u}$ repulsive state has a nonnegligible oscillator strength in the part of the spectrum we are investigating, and would therefore also make a contribution to the Raman scattering intensity. However, the position of this repulsive state is not well known. Although the contribution of the ${ }^{1} \Pi_{1 u}$ state to the resonant Raman intensity appears to be small, a quantitative determination of its optical properties in this frequency region should be possible after including the $B$ discrete states in the summation in Eq. (11). We are currently investigating the possibility of using some simplified calculational procedures $^{42}$ to facilitate this study.

\section{ACKNOWLEDGMENTS}

We thank G. D. Patterson, A. Nitzan, and S. H. Dworetsky for many helpful discussions.

${ }^{1}$ W. Holzer, W. F. Murphy, and H. J. Bernstein, J. Chem. Phys. 52, 399 (1970).

${ }^{2}$ M. Mingardi and S. Siebrand, J. Chem. Phys. 62, 1074 (1975).

${ }^{3} \mathrm{~J}$. Behringer in Molecular Spectroscopy edited by R. F. Barrow, D. A. Long, and D. J. Miller (The Chemical Society, London, 1974), Vol. 2, pp. 100-172.

${ }^{4} \mathrm{~J}$. Behringer, J. Raman Spectrosc. 2, 279 (1974).

${ }^{5}$ M. V. Klein, Phys. Rev. B 8, 919 (1973).

${ }^{6} \mathrm{See}$, for example, J. A. Coxon in Molecular Spectroscopy (The Chemical Society, London, 1973), Vol. 1, pp. 177-228.

${ }^{7}$ A. C. Albrecht, J. Chem. Phys. 34, 1476 (1961).

${ }^{8} \mathrm{~J}$. Tang and A. C. Albrecht in Raman Spectroscopy, edited by H. A. Szymanski (Plenum, New York, 1970), Vol. 2, pp. 33-68.

${ }^{9}$ P. P. Shorygin, Sov, Phys. -Usp. 16, 99 (1973).

${ }^{10} \mathrm{~S}$. D. Silverstein, General Electric Technical Report \# 73CRD195, June 1973; S. D. Silverstein and R. L. St. Peters, Chem. Phys. Lett. 23, 140 (1973); S. D. Silverstein and R. L. St. Peters, Phys. Rev. A 9, 2720 (1974).

${ }^{11} \mathrm{M}$. Jacon and D. Van Labeke, Mol. Phys. 29, 1241 (1975).

${ }^{12}$ Since the initial results of this calculation were reported [P. F. Williams, D. L. Rousseau, and S. H. Dworetsky, Phys. Rev. Lett. 32, 196 (1974)], several other calculations of lifetime responses using a variety of incident pulse shapes have been published and a prior report has been brought to our attention. These include S. D. Silverstein, General Electric Technical Report \#73CRD196, June 1973; J. M. Friedman and R. M. Hochstrasser, Chem. Phys. 6, 155 (1974); J. O. Berg, C. A. Langhoff, and G. W. Robinson, Chem. Phys. Lett. 29, 305 (1974); R. C. Hilborn, Chem.
Phys. Lett. 32, 76 (1975); S. Mukamel and J. Jortner, J. Chem. Phys. 62, 3609 (1975); H. J. Kimble and L. Mandel, Opt. Commun. 14, 167 (1975); H. Metiu, J. Ross, and A. Nitzan, J. Chem. Phys. 63, 1289 (1975).

${ }^{13}$ S. L. McCall, Jr. (private communication).

${ }^{14}$ S. E. Schwartz, Phys. Rev. A 11, 1121 (1975); J. R. Solin and H. Merkelo, Phys. Rev. B 12, 624 (1975).

${ }^{15}$ D. L. Huber, Phys. Rev. 178, 93 (1969).

${ }^{16}$ B. R. Mollow, Phys. Rev. A 2, 76 (1970); B. R. Mollow in Third Rochester Conference on Coherence and Quantum Optics, (Plenum, New York, 1973), pp. 525-532; B. R. Mollow, Phys. Rev. A 8, 1949 (1973); and A. Omont, E. W. Smith, and J. Cooper, Astrophys. J. 115, 185 (1972).

${ }^{17}$ D. L. Huber, Phys. Rev. B 1, 3409 (1970).

${ }^{18}$ Y. R. Shen, Phys. Rev. B 9, 622 (1974).

${ }^{19}$ D. L. Rousseau, G. D. Patterson, and P. F. Williams, Phys. Rev. Lett. 34, 1306 (1975).

${ }^{20}$ P. F. Williams and D. L. Rousseau, Phys, Rev. Lett. 30, 961 (1973).

${ }^{21}$ D. G. Fouche and R. K. Chang, Phys. Rev. Lett. 29, 536 (1972).

${ }^{22} \mathrm{~A}$. Weber, in The Raman Effect (Marcel Dekker, New York, 1973), Vol. 2, pp. 543-759.

${ }^{23}$ W. Kiefer, Appl. Spectrosc. 28, 115 (1974).

${ }^{24}$ R. B. Kurzel, J. I. Steinfeld, D. A. Hatzenbuhler, and G. E. Leroi, J. Chem. Phys. 55, 4822 (1971).

${ }^{25}$ P. F. Williams, D. L. Rousseau, and S. H. Dworetsky, Phys. Rev. Lett. 32, 196 (1974).

${ }^{26}$ G. D. Patterson, S. H. Dworetsky, and R. S. Hozack, J. Mol. Spectrosc. 55, 175 (1975).

${ }^{27}$ G. D. Patterson (private communication).

${ }^{28}$ R. Z. Bachrach, Rev. Sci. Instrum, 43, 734 (1972).

${ }^{29}$ S. Ezekiel and R. Weiss, Phys. Rev. Lett. 20, 91 (1968); and G. A. Capelle and H. P. Broida, J. Chem. Phys. 58, 4212 (1973).

${ }^{31}$ See, for example, K. B. McAfee and R. S. Hozack, J. Chem. Phys. 64, 2491 (1976).

${ }^{32}$ W. Kiefer and H. J. Bernstein, J. Mol. Spectrosc. 43, 366 (1972).

${ }^{33} \mathrm{~J}$. Tellinghuisen, J. Chem. Phys. 58, 2821 (1973).

${ }^{34} \mathrm{~J}$. I. Steinfeld, R. N. Zare, L. Jones, and W. Klemperer, J. Chem. Phys. 42, 25 (1965).

${ }^{35}$ R. N. Zare, J. Chem. Phys. 40, 1934 (1964); R. Verma, J. Chem. Phys. 32, 738 (1960).

${ }^{36} \mathrm{~J}$, W. Cooley, Math. Computation 15, 363 (1961).

${ }^{37} \mathrm{G}$. Herzberg, Molecular Spectra and Molecular Structure (Van Nostrand-Reinhold, New York, 1950), p. 427.

${ }^{38}$ Qualitatively similar calculations were made by M. Berjot, M. Jacon, and L. Bernard [Opt. Commun. 4, 246 (1971)], but they only included contributions from $v^{\prime \prime}=0$ and 1 in their calculations.

${ }^{39}$ G. Placzek and E. Teller, Z. Phys. 81, 209 (1933).

${ }^{40}$ D. L. Rousseau and P. F. Williams, Phys. Rev. Lett. 33, 1368 (1974).

${ }^{41}$ R. J. Oldman, R. K. Sander, and K. R. Wilson, J. Chem. Phys. 54, 4127 (1971).

${ }^{42} \mathrm{~J}$. M. Schulman and R. Detrano, Phys. Rev. A 10, 1192 (1974). 